\title{
Part Two
}

Hybrid Methods

and Models of

Granular

Computing 
OTE/SPH OTE/SPH

JWBK123-22 JWBK123-Pedrycz

February 29, 2008 


\title{
22 \\ A Survey of Interval-Valued Fuzzy Sets
}

\author{
H. Bustince, J. Montero, M. Pagola, E. Barrenechea, and D. Gomez
}

\subsection{Introduction}

Zadeh presented the theory of fuzzy sets in 1965 [1]. From the beginning it was clear that this theory was an extraordinary tool for representing human knowledge. Nevertheless, Zadeh himself established in 1973 (see [2]) that sometimes, in decision-making processes, knowledge is better represented by means of some generalizations of fuzzy sets. The so-called extensions of fuzzy set theory arise in this way.

In the applied field, the success of the use of fuzzy set theory depends on the choice of the membership function that we make. However, there are applications in which experts do not have precise knowledge of the function that should be taken. In these cases, it is appropriate to represent the membership degree of each element to the fuzzy set by means of an interval. From these considerations arises the extension of fuzzy sets called theory of interval-valued fuzzy sets (IVFSs), that is, fuzzy sets such that the membership degree of each element of the fuzzy set is given by a closed subinterval of the interval $[0,1]$. Hence, not only vagueness (lack of sharp class boundaries) but also a feature of uncertainty (lack of information) can be addressed intuitively.

Therefore (see [3]), membership functions of IVFSs are not as specific as their counterparts of fuzzy sets, but this lack of specificity makes them more realistic in some applications. Their advantage is that they allow us to express our uncertainty in identifying a particular membership function. This uncertainty is involved when IVFSs are processed, making results of the processing less specific but more credible.

These sets were born in the 1970s. In May 1975 Sambuc (see [4]) presented in his doctoral thesis the concept of an IVFS named a $\Phi$-fuzzy set. That same year, Jahn [5] wrote about these sets and Zadeh [6] discussed the representation of type 2 fuzzy sets and its potential in approximate reasoning. One year later, Grattan-Guinness [7] established a definition of an interval-valued membership function. In that decade, IVFSs appeared in the literature in various guises and it was not until the 1980s, with the work of Gorzalczany and Turksen [8-16], that the importance of these sets, as well as their name, was definitely established.

In this chapter, we present a survey of IVFSs. We describe the most important concepts of this theory and provide a set of references that is intended to represent as best as possible the work carried out on this extension.

\footnotetext{
Handbook of Granular Computing $\quad$ Edited by Witold Pedrycz, Andrzej Skowron and Vladik Kreinovich
} (C) 2008 John Wiley \& Sons, Ltd 
In this introduction, we must indicate the three major problems, in our opinion, of the theory that is the object of our study:

- A large number of contributions are generalized adaptations of the theoretical developments of fuzzy set theory. This prevents us from focusing on the nature of IVFSs themselves and studying the properties possessed exclusively by these sets.

- In the early work on this theory, Gorzalczany presented the concept of the degree of compatibility between two IVFSs as an interval (see [9]). In some of the latest publications that we have read on interval-valued measures of information, these measures are defined as a point on [0,1]. With this modelization, what is achieved is that the relation between different interval-valued measures of information is a copy of the relation that exists between these concepts in fuzzy set theory. However, we consider that in this case we lose the information that would be provided by a modelization of these measures using intervals.

- Currently, there are two names for these sets: some authors call them interval-valued fuzzy sets and others interval type 2 fuzzy sets. In [17] Mendel writes, 'it turns out that an interval type 2 fuzzy set is the same as an interval-valued fuzzy set for which there is a very extensive literature. These two seemingly different kinds of fuzzy sets were historically approached from very different starting points, which as we shall explain next has turned out to be a very good thing'. Nonetheless, we consider that this duplicity in the name can cause confusion. We have observed that in some papers results that have already been known for many years for IVFSs are presented for interval type 2 fuzzy sets. For this reason, we believe the name should be set; otherwise, the complete bibliography that exists on IVFSs should be taken into account.

Other objections to these sets can be found in [18].

This chapter is organized in the following way. Starting from the definition of an IVFSs and from the study of two construction methods, we present in Section 22.2 the relation between these sets and other representations of fuzzy set theory. Next, in Sections 22.3 and 22.4, we study the connectives and possible combinations between them. In Section 22.5, we analyze the laws for conjunctions and disjunctions. Then, in Section 22.6, we give an interpretation of interval-valued fuzzy operators together with a construction method. In Section 22.7, we recall information measures, and in Section 22.8, we mention the main fields of application of IVFSs . In the final section (Section 22.9), we analyze the use of IVFSs in granular computing.

\subsection{Preliminary Definitions}

In fuzzy set theory, a function $n:[0,1] \rightarrow[0,1]$ such that $n(0)=1, n(1)=0$ that is decreasing is called negation. If a negation is strictly decreasing and continuous, it is called a strict negation. If $n(n(x))=x$ for all $x \in[0,1]$, then $n$ is involutive. A strong negation is a negation that is strict and involutive. In 1979, Trillas [19] characterized strong negations using automorphisms (see also [20]).

In this chapter we shall denote by $\mathcal{F} S s(U)$ the set of all fuzzy sets defined on a finite referential $U$, where $U$ is a non-empty set. Given a fuzzy set $A=\left\{\left(u, \mu_{A}(u)\right) \mid u \in U\right\} \in \mathcal{F} S s(U)$, the expression $A_{n}=\left\{\left(u, n\left(\mu_{A}(u)\right)\right) \mid u \in U\right\}$ will be used here as the complement of the fuzzy set $A$, where $n$ is a negation. We must point out that two distinct notations are most commonly employed in the literature to denote membership functions. In one of them, the membership function of a fuzzy set $A$ is denoted in the way indicated above by the symbol $\mu_{A}$; that is, $\mu_{A}: U \rightarrow[0,1]$. In the other one, the function is denoted by $A$ and has, of course, the same form: $\rightarrow[0,1]$.

We denote by $L([0,1])$ the set of al ntervals of the closed interval $[0,1]$; that is,

$$
L([0,1])=\left\{\mathbf{x}=[\underline{x}, \bar{x}] \mid(\underline{x}, \bar{x}) \in[0,1]^{2} \text { and } \underline{x} \leq \bar{x}\right\} .
$$

$L([0,1])$ is a partially ordered set with respect to the relation $\leq_{L}$ defined in the following way: given $\mathbf{x}, \mathbf{y} \in L([0,1])$,

$$
\mathbf{x} \leq_{L} \mathbf{y} \text { if and only if } \underline{x} \leq \underline{y} \text { and } \bar{x} \leq \bar{y} .
$$


The relation above is transitive and antisymmetric and it expresses the fact that $\mathbf{x}$ links strongly to $\mathbf{y}$, so that $\left(L([0,1]), \leq_{L}\right)$ is a complete lattice (see [21-23]), where the smallest element is $0_{L}=$ $[0,0]$ and the largest is $1_{L}=[1,1]$. Evidently, it is not a linear lattice, for there exist elements that are not comparable. A very interesting study on the arithmetic operations in $L([0,1])$ can be found in [24-28].

Definition 1. An interval-valued fuzzy set $A$ on the universe $U \neq \emptyset$ is a mapping $A: U \rightarrow L([0,1])$.

Obviously, $A(u)=[\underline{A}(u), \bar{A}(u)] \in L([0,1])$ is the membership degree of $u \in U$. We denote by $\mathcal{I} V F S s(U)$ the set of IVFSs on $U$. We denote by $W$ the length of the interval considered.

In [29], two representation theorems and an equivalent classification theorem for IVFSs are presented. Besides the relation $\leq_{L}$ above, other relations (see [30]) on IVFSs have been studied, including among others the following: $\mathbf{x} \preceq \mathbf{y}$ if and only if $\underline{x} \leq y$ and $\bar{y} \leq \bar{x}$.

\subsubsection{Relation to Other Extensions}

The concept of a type 2 fuzzy set was introduced in 1975 by Zadeh [6] as a generalization of an ordinary fuzzy set. Type 2 fuzzy sets are characterized by a fuzzy membership function, that is, the membership value for each element of the set is itself a fuzzy set in $[0,1]$.

Formally, given the referential set $U$, a type 2 fuzzy set is defined as an object $\overline{\bar{A}}$ which has the following form:

$$
\overline{\bar{A}}=\left\{\left(u, x, \mu_{u}(x)\right) \mid u \in U, x \in[0,1]\right\},
$$

where $x \in[0,1]$ is the primary membership degree of $u$ and $\mu_{u}(x)$ is the secondary membership level, specific to a given pair $(u, x)$.

IVFSs can be generalized by assigning to each interval a fuzzy set defined on the referential set $[0,1]$; that is, IVFSs can be generalized by means of type 2 fuzzy sets. The following equation shows a way of constructing a type 2 fuzzy set from an $A \in \operatorname{IVFSs}(U)$ :

$$
\mu_{u}(x)= \begin{cases}0 & \text { if } 0 \leq x \leq \underline{A}(u) \\ \frac{2}{\bar{A}(u)-\underline{A}(u)}(x-\underline{A}(u)) & \text { if } \underline{A}(u) \leq x \leq \underline{\underline{A}(u)+\bar{A}(u)} \\ \frac{2}{2}(x-\bar{A}(u)) & \text { if } \frac{\underline{A}(u)+\bar{A}(u)}{2} \leq x \leq \bar{A}(u) \\ \frac{\bar{A}(u)-\bar{A}(u)}{0} & \text { if } \bar{A}(u) \leq x \leq 1 .\end{cases}
$$

Evidently, the choice of a triangular shape for the membership function is totally arbitrary; we can associate another type of membership function, for example, a trapezoidal shape, with the set.

A particular case of a type 2 fuzzy set is an interval type 2 fuzzy set (see [17, 31-17]). An interval type 2 fuzzy set $A$ in $U$ is defined by

$$
\overline{\bar{A}}=\left\{\left(u, A(u), \mu_{u}(x)\right) \mid u \in U, A(u) \in L([0,1])\right\},
$$

where $A(u)$ is the membership function presented in Definition 1; that is, it is a closed subinterval of $[0,1]$, and the function $\mu_{u}(x)$ represents the fuzzy set associated with the element $u \in U$ obtained when $x$ covers the interval $[0,1] ; \mu_{u}(x)$ is given in the following way:

$$
\mu_{u}(x)= \begin{cases}a & \text { if } \underline{A}(u) \leq x \leq \bar{A}(u) \\ 0 & \text { otherwise }\end{cases}
$$

where $0 \leq a \leq 1$. In [17,31-33], it turns out that an interval type 2 fuzzy set is the same as an IVFS if we take $a=1$. 
Another important extension of fuzzy set theory is the theory of Atanassov's intuitionistic fuzzy sets (A-IFSs) $[34,35]$. A-IFSs assign to each element of the universe not only a membership degree, but also a non-membership degree, which is less than or equal to 1 minus the membership degree.

An A-IFS on $U$ is a set

$$
A=\left\{\left(u, \mu_{A}(u), v_{A}(u)\right) \mid u \in U\right\},
$$

where $\mu_{A}(u) \in[0,1]$ denotes the membership degree and $v_{A}(u) \in[0,1]$ the non-membership degree of $u$ in $A$ and where, for all $u \in U, \mu_{A}(u)+v_{A}(u) \leq 1$.

In [34], Atanassov established that every Atanassov's intuitionistic fuzzy set $A$ on $U$ can be represented by an interval-valued fuzzy set $A$ given by

$$
\begin{aligned}
A: & U \rightarrow L([0,1]) \\
& u \rightarrow\left[\mu_{A}(u), 1-v_{A}(u)\right], \quad \text { for all } u \in U .
\end{aligned}
$$

Using this representation, Atanassov proposed in 1983 that A-IFS theory was equivalent to the theory of IVFSs. This equivalence was proved in 2003 by Deschrijver and Kerre [23]. Therefore, from a mathematical point of view, the results that we obtain for IVFSs are easily adaptable to A-IFSs and vice versa. Nevertheless, we need to point out that, conceptually, the two types of sets are totally different. This is made clear when applications of these sets are constructed (see [36-39]).

In 1993, Gau and Buehrer introduced the concept of vague sets [40]. Later, in 1996, it was proved that vague sets are in fact A-IFSs [41].

A compilation of the sets that are equivalent (from a mathematical point of view) to IVFSs can be found in [42]. Two conclusions are drawn from this study:

1. IVFSs are equivalent to A-IFSs (and therefore vague sets), to grey sets (see [43]), and to $L$-fuzzy set in Goguen's sense (see $[44,45])$ with respect to a special lattice $L([0,1])$.

2. IVFSs are a particular case of probabilistic sets (see [46]), of soft sets (see [47]), of Atanassov's interval-valued intuitionistic fuzzy sets (see [35]) and evidently of type 2 fuzzy sets.

\subsubsection{Some Methods of Construction of Interval-Valued Fuzzy Sets}

In 1975 Sambuc used IVFSs for the construction of a computer system that would help make a diagnosis of certain thyroidal pathologies. In this paper, the problem of constructing an interval-valued membership function that is more appropriate for the computer system arises for the first time.

Later, Turksen and Yao (see [16]) proposed a method of constructing IVFSs using the fact that in fuzzy logic it generally occurs that the conjunctive normal form (CNF) gives membership grades greater than the disjunctive normal form (DNF). Ten years later, Turksen (see $[13,48]$ ) proved that when performing a fuzzy set-theoretic aggregation of regular membership functions, the simultaneous use of CNF and DNF forms of the fuzzy connectives yields an IVFSs.

The constructions of IVFSs presented in 1996 (see [49]) are divided into two groups: constructions from a fuzzy set and constructions from two or more fuzzy sets. Next we present an example of each of them.

\section{Construction of an IVFSs from a Fuzzy Set}

In 2005 Tizhoosh (see [50]) used IVFSs for determining the threshold of an image $Q$. The threshold enables to separate the object contained in the image from its background.

In his work, Tizhoosh associates each image with an IVFSs in the following way:

1. Have an expert assign the image a fuzzy set characterized by the membership function $\mu_{Q}$.

2. For each pixel, construct the interval $\left[\left(\mu_{Q}\right)^{\alpha},\left(\mu_{Q}\right)^{\frac{1}{\alpha}}\right]$ with $\alpha \in(1, \infty)$, which represents its membership to the IVFS that is going to represent the image (see Figure 22.1).

The relevance of this construction has been proved in an experimental way in [50-52]. In these works one concludes that in images with many pixels for which experts do not agree on whether they belong 


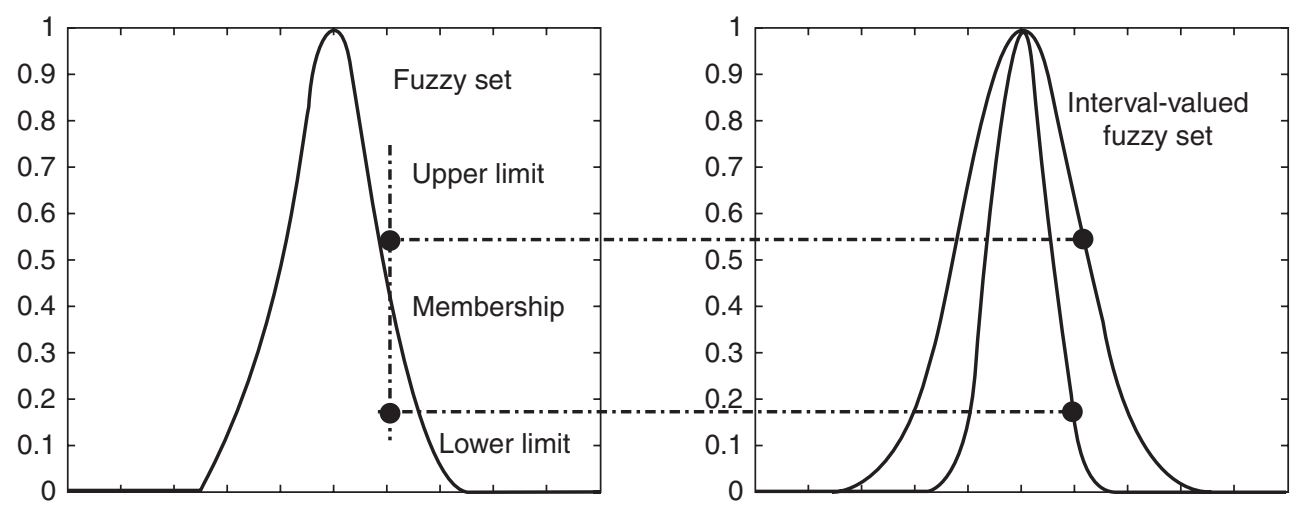

Figure 22.1 Method of construction of an IVFS from a fuzzy set

to the background or to the object, the thresholds obtained with these constructions (together with the algorithm that we will describe in Section 22.8) are much better than those obtained with classical methods or with methods that use fuzzy techniques.

A generalization of this method can be found developed in [53].

\section{Construction of an IVFSs from Two or More Fuzzy Sets}

If we ask several experts to construct the membership function that represents the fuzzy set that modelizes a certain action, we discover the fact that in most cases the experts choose different membership functions. Because of this we have uncertainty in choosing the best function. In these conditions it is recommended to work with IVFSs constructed in the following way: each element is assigned an interval whose lower extreme is the lowest value given by the experts for that element and as upper extreme the highest (see [54-57]).

Other construction methods can be found in $[38,58,59]$.

\subsubsection{A Method of Construction of Fuzzy Sets from Interval-Valued Fuzzy Sets}

The operator that we present next will enable us to construct a family of fuzzy sets from an IVFS (see [60]). This concept will be frequently used throughout the whole chapter.

Definition 2. Let $\alpha \in[0,1]$; we define $K_{\alpha}$ as a function $K_{\alpha}: L([0,1]) \rightarrow[0,1]$ such that it satisfies the following conditions:

1. If $\underline{x}=\bar{x}$, then $K_{\alpha}(\mathbf{x})=\underline{x}$.

2. $K_{0}(\mathbf{x})=\underline{x}, K_{1}(\mathbf{x})=\bar{x}$ for all $\mathbf{x} \in L([0,1])$.

3. If $\mathbf{x} \leq_{L} \mathbf{y}$, with $\mathbf{x}, \mathbf{y} \in L([0,1])$, then $K_{\alpha}(\mathbf{x}) \leq K_{\alpha}(\mathbf{y})$ for all $\alpha \in[0,1]$.

4. $K_{\alpha}(\mathbf{x}) \leq K_{\beta}(\mathbf{x})$ if and only if $\alpha \leq \beta$, for all $\mathbf{x} \in L([0,1])$, with $\beta \in[0,1]$.

A study of these operators can be found in [35] (and also in [49, 61]). In these papers, the following expressions are proposed for these operators:

$$
K_{\alpha}(\mathbf{x})=K_{\alpha}([\underline{x}, \bar{x}])=K_{\alpha}\left(\left[K_{0}(\mathbf{x}), K_{1}(\mathbf{x})\right]\right)=\underline{x}+\alpha(\bar{x}-\underline{x})=K_{0}(\mathbf{x})+\alpha W_{\mathbf{x}},
$$

where $W_{\mathbf{X}}$ represents the length of the interval $\mathbf{x}$. 
The operator $K_{\alpha}$ enables every IVFS to be associated with a fuzzy set in the following way (see [21, $35,41,49,53,54,61-66])$ :

$$
K_{\alpha}: \mathcal{I V F S s}(U) \rightarrow \mathcal{F} S s(U)
$$

given by

$$
K_{\alpha}(A)=\left\{\left(u, \mu_{K_{\alpha}(A)}(u)=K_{\alpha}(A(u))=K_{\alpha}([\underline{A}(u), \bar{A}(u)]) \mid u \in U\right\}\right.
$$

Unless otherwise indicated, the operator $K_{\alpha}$ that we shall use is the general operator presented in Definition 2. That is, we shall not use any particular expression for it.

\subsection{Connectives}

In various papers (e.g., [4, 9, 21, 35, 41, 47, 49, 51-54, 60-66, 68-82]), the union, intersection, and complementation of $\mathcal{I V F S s}(U)$ are defined in the following way: if $A, B \in \mathcal{I V F S s}(U)$, then

$$
\begin{aligned}
A \cap B(u) & =\left[\min \left(K_{0}(A(u)), K_{0}(B(u))\right), \min \left(K_{1}(A(u)), K_{1}(B(u))\right)\right], \\
A \cup B(u) & =\left[\max \left(K_{0}(A(u)), K_{0}(B(u))\right), \max \left(K_{1}(A(u)), K_{1}(B(u))\right)\right], \\
A_{n}(u) & =\left[1-K_{1}(A(u)), 1-K_{0}(A(u))\right] \text { for all } u \in U .
\end{aligned}
$$

In $[21,64,65]$, it is proved that $\{I V F S s, \cap, \cup\}$ is a distributive, bounded, non-complemented lattice that satisfies De Morgan's laws (with respect to the definition of $A_{n}$ above).

Next, in the first subsection we thoroughly study the concept of interval-valued negation and in subsequent subsections we describe the manner of modelizing the operations of union and intersection by means $t$-norms and $t$-conorms.

\subsubsection{Negations}

Interval-valued negations, hereinafter referred to as IV negations, are an extension of negations and are defined as follows:

Definition 3. An IV negation is a function $N: L([0,1]) \rightarrow L([0,1])$ that is decreasing (with respect to $\left.\leq_{L}\right)$ such that $N\left(1_{L}\right)=0_{L}$ and $N\left(0_{L}\right)=1_{L}$. If for all $\mathbf{x} \in L([0,1]), N(N(\mathbf{x}))=\mathbf{x}$, it is said that $N$ is involutive.

Next, we present a theorem related to the construction of IV negations. Historically, this theorem provided the first construction method for such negations (see [21, 53]).

Theorem 1. Let the function $N: L([0,1]) \rightarrow L([0,1])$ be given by

$$
N(\mathbf{x})=\left[n\left(K_{1}(\mathbf{x})\right), n\left(K_{0}(\mathbf{x})\right)\right]
$$

where $n:[0,1] \rightarrow[0,1]$ is a negation. Under these conditions $N$ is an IV negation.

An in-depth study of strict IV negations can be found in [54, 77].

\section{Representation Theorem for IV Negations}

A representation theorem for IV negations was obtained in [54]. Previously Deschrijver et al. (see [77]) proved this theorem for A-IFSs. The only small difference between these two studies lies essentially in the fact that for the negations of the A-IFSs, $K_{\alpha}$ operators are not used, whereas these operators are used 
in the characterization of the IV negations developed in [54]. This fact enables us to prove the following lemma (which is a consequence of Lemma 3.5 proved in [77]):

Lemma 1. Let there be a $K_{\alpha}$ with $\alpha \in[0,1]$. If $N$ is an involutive IV negation, then for every $\mathbf{x}=$ $\left[K_{0}(\mathbf{x}), K_{0}(\mathbf{x})\right] \in L([0,1])$,

$$
K_{0}(N(\mathbf{x}))=K_{\alpha}(N(\mathbf{x}))=K_{1}(N(\mathbf{x}))
$$

holds for all $\alpha \in[0,1]$.

Lemma 1 , together with other properties (see [21, 49, 54,61]) of the operators $K_{\alpha}$, has made it possible to prove the following characterization theorem (which is an adaptation for IVFSs of Theorem 3.6 proved in [77]).

Theorem 2. A function $N: L([0,1]) \rightarrow L([0,1])$ is an involutive IV negation if and only if there exists an involutive negation $n$ such that

$$
N(\mathbf{x})=\left[n\left(K_{1}(\mathbf{x})\right), n\left(K_{0}(\mathbf{x})\right)\right]
$$

\section{Complement of an Interval-Valued Fuzzy Set}

Given an interval-valued fuzzy set $A \in \mathcal{I} V F S s(U)$, from the functions $N$ we can define the concept of the complement of $A$ in the following way:

$$
A_{N}(u)=N(A(u)) \text { for all } u \in U .
$$

\subsubsection{IV t-Norms and IV t-Conorms}

In fuzzy set theory, $t$-norms are used for modeling the intersection (or conjunction) of two fuzzy sets and $t$-conorms for modeling the union (or disjunction). Similarly, in the theory of IVFSs, we can model the intersection and the union using interval-valued $t$-norms and interval-valued $t$-conorms in the following way: for all $u \in U$ and $A, B \in \mathcal{I} \operatorname{VFSs}(U)$, we have

$$
\begin{aligned}
& A \cap_{\mathbf{T}} B(u)=\mathbf{T}(A(u), B(u)), \\
& A \cup_{\mathbf{S}} B(u)=\mathbf{S}(A(u), B(u)),
\end{aligned}
$$

where $\mathbf{T}$ and $\mathbf{S}$ are, according to Definition 4 , an IV $t$-norm and an IV $t$-conorm, respectively (see [60, $65,74])$.

Definition 4. A function $\mathbf{T}:(L([0,1]))^{2} \rightarrow L([0,1])$ is said to be an interval-valued $t$-norm (IV t-norm) if it is commutative, associative, and increasing (in both arguments with respect to the order $\leq_{L}$ ) and has a neutral element $1_{L}=[1,1]$. In the same way, a function $\mathbf{S}:(L([0,1]))^{2} \rightarrow L([0,1])$ is said to be an interval-valued t-conorm (IV t-conorm) if it is commutative, associative, and increasing and has a neutral element $0_{L}=[0,0]$.

Evidently, $\mathbf{T}\left(\mathbf{x}, 0_{L}\right) \leq_{L} \mathbf{T}\left(1_{L}, 0_{L}\right)=0_{L}$, and therefore $\mathbf{T}\left(\mathbf{x}, 0_{L}\right)=0_{L}$. In a similar way, we have $\mathbf{S}\left(\mathbf{x}, 1_{L}\right)=1_{L}$. Note that Definition 4 is an extension of the classical definition of the $t$-norm and $t$-conorm in $[0,1]$. We only need to substitute $L([0,1])$ for $[0,1]$.

A lot has been written on the way in which IV $t$-norms and IV $t$-conorms can be generated from $t$-norms and $t$-conorms in $[0,1]$. The first idea that appeared was the generation of IV $t$-norms (and IV $t$-conorms) from two $t$-norms $T_{a}$, and $T_{b}$ (and $S_{a}$, and $S_{b}$ ) in [0,1], from the operator $K_{\alpha}$ and from expressions in the following way (see $[60,65])$ :

$$
\left\{\begin{array}{l}
\mathbf{T}(\mathbf{x}, \mathbf{y})=\left[T_{a}\left(K_{\alpha}(\mathbf{x}), K_{\alpha}(\mathbf{y})\right), T_{b}\left(K_{\beta}(\mathbf{x}), K_{\beta}(\mathbf{y})\right)\right] \\
\mathbf{S}(\mathbf{x}, \mathbf{y})=\left[S_{a}\left(K_{\alpha}(\mathbf{x}), K_{\alpha}(\mathbf{y})\right), S_{b}\left(K_{\beta}(\mathbf{x}), K_{\beta}(\mathbf{y})\right)\right] .
\end{array}\right.
$$

A justification for the choice of these expressions and a demonstration of the following theorem can be found in [54]. 
Theorem 3. (a) Let $\alpha, \beta \in[0,1]$ such that $\alpha<\beta$ and let $T_{a}$ and $T_{b}$ be two t-norms in $[0,1]$ such that $T_{a} \leq T_{b}$. Let the function $\mathbf{T}:(L([0,1]))^{2} \rightarrow L([0,1])$ be given by

$$
\mathbf{T}(\mathbf{x}, \mathbf{y})=\left[T_{a}\left(K_{\alpha}(\mathbf{x}), K_{\alpha}(\mathbf{y})\right), T_{b}\left(K_{\beta}(\mathbf{x}), K_{\beta}(\mathbf{y})\right)\right] \text { for all } \mathbf{x}, \mathbf{y} \in L([0,1]) .
$$

Under these conditions,

$$
\mathbf{T} \text { is an IV t-norm if and only if }\left\{\begin{array}{l}
K_{\alpha}(\mathbf{x})=K_{0}(\mathbf{x}) \\
K_{\beta}(\mathbf{x})=K_{1}(\mathbf{x})
\end{array}\right.
$$

for all $\mathbf{x} \in L([0,1])$.

(b) Let $\alpha, \beta \in[0,1]$ such that $\alpha<\beta$ and let $S_{a}$ and $S_{b}$ be two t-conorms in $[0,1]$ such that $S_{a} \leq S_{b}$. Let the function $\mathbf{S}:(L([0,1]))^{2} \rightarrow L([0,1])$ be given by

$$
\mathbf{S}(\mathbf{x}, \mathbf{y})=\left[S_{a}\left(K_{\alpha}(\mathbf{x}), K_{\alpha}(\mathbf{y})\right), S_{b}\left(K_{\beta}(\mathbf{x}), K_{\beta}(\mathbf{y})\right)\right] \text { for all } \mathbf{x}, \mathbf{y} \in L([0,1]) .
$$

Under these conditions,

$$
\mathbf{S} \text { is an IV t-conorm if and only if }\left\{\begin{array}{l}
K_{\alpha}(\mathbf{x})=K_{0}(\mathbf{x}) \\
K_{\beta}(\mathbf{x})=K_{1}(\mathbf{x})
\end{array}\right.
$$

for all $\mathbf{x} \in L([0,1])$

A consequence of Theorem 3 is the following corollary (see [22, 23, 43, 51, 52, 60, 64-66, 68-77]). This corollary provides a construction method for IV $t$-norms and IV $t$-conorms, which was presented in the first papers on this topic.

Corollary 1. (a) If $T_{a}$ and $T_{b}$ are two t-norms in $[0,1]$ such that $T_{a}(x, y) \leq T_{b}(x, y)$ for all $x, y \in[0,1]$, then the function $\mathbf{T}:(L([0,1]))^{2} \rightarrow L([0,1])$ defined for each $\mathbf{x}, \mathbf{y} \in L([0,1])$ by

$$
\mathbf{T}(\mathbf{x}, \mathbf{y})=\left[T_{a}\left(K_{0}(\mathbf{x}), K_{0}(\mathbf{y})\right), T_{b}\left(K_{1}(\mathbf{x}), K_{1}(\mathbf{y})\right)\right]
$$

is an IV t-norm.

(b) If $S_{a}$ and $S_{b}$ are two t-conorms in $[0,1]$ such that $S_{a}(x, y) \leq S_{b}(x, y)$ for all $x, y \in[0,1]$, then the function $\mathbf{S}:(L([0,1]))^{2} \rightarrow L([0,1])$ defined for each $\mathbf{x}, \mathbf{y} \in L([0,1])$ by

$$
\mathbf{S}(\mathbf{x}, \mathbf{y})=\left[S_{a}\left(K_{0}(\mathbf{x}), K_{0}(\mathbf{y})\right), S_{b}\left(K_{1}(\mathbf{x}), K_{1}(\mathbf{y})\right)\right]
$$

is an IV t-conorm.

The following theorem, proved in [54], makes it clear that the converse of Corollary 1 is not true: that is, there are IV $t$-norms (and IV $t$-conorms) that are not generated from expressions of the type (1).

Theorem 4. (a) Let there be an operator $K_{\alpha}$ with $\alpha \in[0,1]$ and let $T$ be any $t$-norm in $[0,1]$. Let there be a function

$$
\mathbf{T}_{\alpha}^{T}(\mathbf{x}, \mathbf{y})=\left\{\begin{array}{l}
\mathbf{x} \text { if } \mathbf{y}=1_{L} \\
\mathbf{y} \text { if } \mathbf{x}=1_{L} \\
{\left[T\left(K_{\alpha}(\mathbf{x}), K_{\alpha}(\mathbf{y})\right), T\left(K_{\alpha}(\mathbf{x}), K_{\alpha}(\mathbf{y})\right)\right]}
\end{array}\right.
$$

Under these conditions,

$$
\mathbf{T}_{\alpha}^{T} \text { is an IVt-norm if and only if } K_{\alpha}(\mathbf{x})=K_{0}(\mathbf{x}) \text { for all } \mathbf{x} \in L([0,1]) .
$$

(b) Let there be an operator $K_{\alpha}$ with $\alpha \in[0,1]$ and let $S$ be any $t$-conorm in $[0,1]$. Let there be a function

$$
\mathbf{S}_{\alpha}^{S}(\mathbf{x}, \mathbf{y})=\left\{\begin{array}{l}
\mathbf{x} \text { if } \mathbf{y}=0_{L} \\
\mathbf{y} \text { if } \mathbf{x}=0_{L} \\
{\left[S\left(K_{\alpha}(\mathbf{x}), K_{\alpha}(\mathbf{y})\right), S\left(K_{\alpha}(\mathbf{x}), K_{\alpha}(\mathbf{y})\right)\right] .}
\end{array}\right.
$$




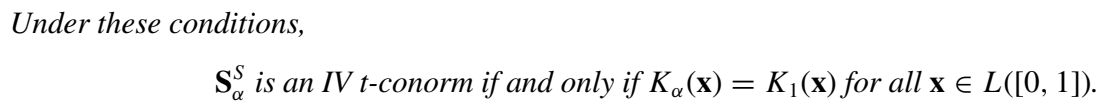

$$
\mathbf{S}_{\alpha}^{S} \text { is an IV t-conorm if and only if } K_{\alpha}(\mathbf{x})=K_{1}(\mathbf{x}) \text { for all } \mathbf{x} \in L([0,1]) \text {. }
$$

From now on, we shall denote $\mathbf{T}_{0}^{T}$ and $\mathbf{S}_{1}^{S}$ by $\mathbf{T}^{T}$ and $\mathbf{S}^{S}$, respectively.

\section{t-Representable IV t-norms and s-Representable IV t-Conorms}

The objective of constructing characterization theorems for IV $t$-norms (and IV $t$-conorms) led Cornelis et al. [74] and Deschrijver et al. [77] to introduce the concepts of $t$-representable IV $t$-norm and $s$-representable IV $t$-conorm.

Definition 5. (a) An IV t-norm is said to be t-representable if there are two t-norms $T_{a}$ and $T_{b}$ in $[0,1]$ such that $\mathbf{T}(\mathbf{x}, \mathbf{y})=\left[T_{a}\left(K_{0}(\mathbf{x}), K_{0}(\mathbf{y})\right), T_{b}\left(K_{1}(\mathbf{x}), K_{1}(\mathbf{y})\right)\right]$ for all $\mathbf{x} \in L([0,1])$ and for all $\mathbf{y} \in L([0,1])$, such that $\mathbf{x}=\left[K_{0}(\mathbf{x}), K_{1}(\mathbf{x})\right], \mathbf{y}=\left[K_{0}(\mathbf{y}), K_{1}(\mathbf{y})\right]$.

(b) An IV t-conorm is said to be s-representable if there are two t-conorms $S_{a}$ and $S_{b}$ in $[0,1]$ such that $\mathbf{S}(\mathbf{x}, \mathbf{y})=\left[S_{a}\left(K_{0}(\mathbf{x}), K_{0}(\mathbf{y})\right), S_{b}\left(K_{1}(\mathbf{x}), K_{1}(\mathbf{y})\right)\right]$ for all $\mathbf{x} \in L([0,1])$ and for all $\mathbf{y} \in L([0,1])$, such that $\mathbf{x}=\left[K_{0}(\mathbf{x}), K_{1}(\mathbf{x})\right], \mathbf{y}=\left[K_{0}(\mathbf{y}), K_{1}(\mathbf{y})\right]$.

We denote by $\mathbf{T}_{T_{a}, T_{b}}$ the $t$-representable IV $t$-norms obtained by means of the $t$-norms $T_{a}$ and $T_{b}$ in $[0,1]$. We denote similarly the $s$-representable IV $t$-conorms $\mathbf{S}_{S_{a}, S_{b}}$.

Some examples of $t$-representable IV $t$-norms and $s$-representable IV $t$-conorms are the ones studied in Theorem 3 Some examples of non- $t$-representable IV $t$-norms and non- $s$-representable IV $t$-conorms are the ones studied in Theorem 4 (see [77]).

In [23, 43, 72-77], it is proved that some important representation theorems can be also shown for IV $t$-norms, but not for $t$-representable $t$-norms. This shows that IVFS theory cannot be reduced to an approach in which all operators are $t$-representable. In any case, the theoretical developments described in these publications are similar (although in a certain way they generalize them) to the theoretical developments carried out in [3, 20, 82] in order to characterize $t$-norms (and $t$-conorms) in [0,1]. In [79], the concept of the interval-valued uninorm was analyzed for the first time.

\section{The Archimedean Property of IV t-Norms}

Starting from the work on $t$-norms in [0,1] of Klement et al. (see [83-86]), Deschrijver adapted the Archimedean property of $t$-norms in [0,1] to IV $t$-norms in [78]. In that publication, new and important concepts of weak Archimedean and strong Archimedean properties were presented, and the definition of the pseudo-t-representable IV t-norm was introduced in the following way:

An IV $t$-norm is called pseudo-t-representable if there exists a $t$-norm $T$ on $([0,1], \leq)$ such that, for all $\mathbf{x}, \mathbf{y} \in L([0,1])$,

$$
\mathcal{T}(\mathbf{x}, \mathbf{y})=\left[T\left(K_{0}(\mathbf{x}), K_{0}(\mathbf{y})\right), \max \left(T\left(K_{0}(\mathbf{x}), K_{1}(\mathbf{y})\right), T\left(K_{1}(\mathbf{x}), K_{0}(\mathbf{y})\right)\right)\right]
$$

In [78], there is a study of the conditions under which $t$-representable IV $t$-norms and pseudo- $t$ representable IV $t$-norms satisfy the Archimedean property, the weak Archimedean property, or the strong Archimedean property. Particularly it is proved that the pseudo- $t$-representable IV $t$-norms satisfy the Archimedean property but the $t$-representable IV $t$-norms do not. This also justifies the comment above regarding the fact that IVFS theory cannot be reduced to an approach in which all operators are $t$-representable.

\subsection{Combinations of Operations}

Theorem 5. Let $N$ be any involutive IV negation. The following items hold:

(a) If $\mathbf{T}$ is an IV $t$-norm, then the function defined by $\mathbf{S}^{*}(\mathbf{x}, \mathbf{y})=N(\mathbf{T}(N(\mathbf{x}), N(\mathbf{y})))$ for all $\mathbf{x}, \mathbf{y} \in L([0,1])$ is an IV t-conorm. 
(b) If $\mathbf{T}$ is t-representable, then $\mathbf{S}^{*}$ is s-representable.

(c) If $\mathbf{S}$ is an IV t-conorm, then the function defined by

$\mathbf{T}^{*}(\mathbf{x}, \mathbf{y})=N(\mathbf{S}(N(\mathbf{x}), N(\mathbf{x})))$ for all $\mathbf{x}, \mathbf{y} \in L([0,1])$ is an IV t-norm.

(d) If $\mathbf{S}$ is s-representable, then $\mathbf{T}^{*}$ is t-representable.

Given an IV $t$-norm $\mathbf{T}$, we call the expression for item (a) of Theorem 5 ,

$$
\mathbf{S}^{*}(\mathbf{x}, \mathbf{y})=N(\mathbf{T}(N(\mathbf{x}), N(\mathbf{y}))) \text { for all } \mathbf{x}, \mathbf{y} \in L([0,1]),
$$

the dual IV $t$-conorm of $\mathbf{T}$ with respect to the IV negation $N$. Similarly, we call the expression for $\mathbf{T}^{*}$ in item (c) the dual IV $t$-norm of the IV $t$-conorm $\mathbf{S}$ with respect to the IV negation $N$ [77].

Let the triple ( $\mathbf{T}, \mathbf{S}, N)$ (where $N$ is an involutive IV negation) denote that $\mathbf{T}$ and $\mathbf{S}$ are dual with respect to $N$; any such triple is called a dual triple sometimes also called a De Morgan triple (see [54, 77] and Section 4 in [84]).

\subsection{Laws for Conjunctions and Disjunctions}

In this section, we set out to study properties of set theory such as idempotency, absorption, distributiveness, the law of contradiction, and the law of the excluded middle. These properties must be defined for IVFSs. We start from the work of Jenei [30] (see also [54, 76]) and we analyze the conditions under which IV $t$-norms and IV $t$-conorms satisfy or do not satisfy these properties. Some of the theorems that we present in this section are proved in [54] and others in [30].

\subsubsection{Law of Contradiction and Law of the Excluded Middle}

Here, we study the conditions under which the law of contradiction and the law of the excluded middle are satisfied for IVFSs. For this purpose, we understand the law of contradiction for IVFSs in this manner, analogously to the cases of classical and fuzzy sets: for all $\mathbf{x} \in L([0,1])$, it should hold that $\mathbf{T}(\mathbf{x}, N(\mathbf{x}))=0_{L}$. Similarly, the law of the excluded middle says that for all $\mathbf{x} \in L([0,1])$, it should hold that $\mathbf{S}(\mathbf{x}, N(\mathbf{x}))=1_{L}$.

Theorem 6. The following items hold:

(a) If $\mathbf{T}_{T_{a}, T_{b}}$ is a t-representable IV t-norm, then for any involutive IV negation, the law of contradiction is not satisfied.

(b) If $\mathbf{S}_{S_{a}, S_{b}}$ is an s-representable IV t-conorm, then for any involutive IV negation, the law of the excluded middle is not satisfied.

It is necessary to point out that there exist IV $t$-norms and IV $t$-conorms that are not $t$-representable or $s$-representable and satisfy the law of contradiction and the law of the excluded middle, respectively. This fact is made clear in the following theorem.

Theorem 7. (a) Let there be a t-norm $T$ in $[0,1]$ that satisfies the law of contradiction with respect to the involutive negation $n$ and let the IV t-norm $\mathbf{T}^{T}$ be generated by that $t$-norm. Then $\mathbf{T}^{T}$ satisfies the law of contradiction with respect to the involutive IV negation $N$ generated by $n$.

(b) Let there be a t-conorm $S$ in $[0,1]$ that satisfies the law of the excluded middle with respect to the involutive negation $n$ and let the IV t-conorm $\mathbf{S}^{S}$ be generated by that t-conorm. Then $\mathbf{S}^{S}$ satisfies the law of the excluded middle with respect to the involutive IV negation $N$ generated by $n$.

From Theorem 7 we deduce that there exist non- $t$-representable IV $t$-norms that satisfy the law of contradiction; evidently, we can also deduce that there exist non- $s$-representable IV $t$-conorms that satisfy the law of the excluded middle. Nevertheless, it is important to indicate that there exist non- $t$-representable IV $t$-norms that do not satisfy the law of contradiction, and there also exist non-s-representable IV 


\begin{abstract}
$t$-conorms that do not satisfy the law of the excluded middle; specific examples of these IV $t$-norms and IV $t$-conorms can be found in [54]. In that work there are also theorems that establish the manner in which one can construct from automorphisms non-t-representable IV $t$-norms that satisfy the law of contradiction (and similarly for non-s-representable IV $t$-conorms and the law of the excluded middle).
\end{abstract}

\title{
22.5.2 Idempotency, Absorption, and Distributivity
}

Theorem 8. The following items hold:
(a) $\mathbf{T}(\mathbf{x}, \mathbf{x})=\mathbf{x}$ for all $\mathbf{x} \in L([0,1])$ (i.e., $\mathbf{T}$ is idempotent) if and only if $\mathbf{T}=\mathbf{T}_{\text {min,min }}$.
(b) $\mathbf{S}(\mathbf{x}, \mathbf{x})=\mathbf{x}$ for all $\mathbf{x} \in L([0,1])$ (i.e., $\mathbf{S}$ is idempotent) if and only if $\mathbf{S}=\mathbf{S}_{\max , \max }$.
(c) $\mathbf{T}(\mathbf{x}, \mathbf{S}(\mathbf{x}, \mathbf{y}))=\mathbf{x}$ for all $\mathbf{x}, \mathbf{y} \in L([0,1])$ (property of absorption) if and only if $\mathbf{T}$ is idempotent.
(d) $\mathbf{S}(\mathbf{x}, \mathbf{T}(\mathbf{x}, \mathbf{y}))=\mathbf{x}$ for all $\mathbf{x}, \mathbf{y} \in L([0,1])$ (property of absorption) if and only if $\mathbf{S}$ is idempotent.

A consequence of Theorem 8 is the following: the algebraic structure $\{$ IVFSs, $\mathbf{T}, \mathbf{S}\}$ is only a lattice when we take $\mathbf{T}=\mathbf{T}_{\text {min, } \min }$ and $\mathbf{S}=\mathbf{S}_{\max , \max }$.

Theorem 9. Let $\mathbf{T}_{T_{a}, T_{b}}$ be a t-representable IV t-norm and let $\mathbf{S}_{S_{a}, S_{b}}$ be an s-representable IV t-conorm. Under these conditions, the following items hold:

(a) $\mathbf{S}_{S_{a}, S_{b}}\left(\mathbf{x}, \mathbf{T}_{T_{a}, T_{b}}(\mathbf{y}, \mathbf{z})\right)=\mathbf{T}_{T_{a}, T_{b}}\left(\mathbf{S}_{S_{a}, S_{b}}(\mathbf{x}, \mathbf{y}), \mathbf{S}_{S_{a}, S_{b}}(\mathbf{x}, \mathbf{z})\right)$ for all $\mathbf{x}, \mathbf{y}, \mathbf{z} \in L([0,1])$, if and only if $\mathbf{T}_{T_{a}, T_{b}}=\mathbf{T}_{\text {min,min }}$.

(b) $\mathbf{T}_{T_{a}, T_{b}}\left(\mathbf{x}, \mathbf{S}_{S_{a}, S_{b}}(\mathbf{y}, \mathbf{z})\right)=\mathbf{S}_{S_{a}, S_{b}}\left(\mathbf{T}_{T_{a}, T_{b}}(\mathbf{x}, \mathbf{y}), \mathbf{T}_{T_{a}, T_{b}}(\mathbf{x}, \mathbf{z})\right)$ for all $\mathbf{x}, \mathbf{y}, \mathbf{z} \in L([0,1])$, if and only if $\mathbf{S}_{S_{a}, S_{b}}=\mathbf{S}_{\text {max }, \text { max }}$.

By Theorems 8 and 9 we have on the one hand the following result: $\left\{I V F S s, \mathbf{T}_{\min , \min }, \mathbf{S}_{\max , \max }\right\}$ is a distributive lattice, and on the other, the following corollary.

Corollary 2. Let $\mathbf{T}_{T_{a}, T_{b}}$ be a t-representable IV t-norm and let $\mathbf{S}_{S_{a}, S_{b}}$ be an s-representable IV t-conorm. Under these conditions, we have distributiveness if and only if we have absorption, if and only if we have idempotency, and

$$
\text { if and only if } \mathbf{T}_{T_{a}, T_{b}}=\mathbf{T}_{\min , \min } \text { and } \mathbf{S}_{S_{a}, S_{b}}=\mathbf{S}_{\max , \max } .
$$

Regarding the distributive property of non-t-representable IV $t$-norms and non- $s$-representable IV $t$-conorms, there exist situations in which this property does not hold, as we will see in the following theorem which is an immediate consequence of this: if $(\mathbf{T}, \mathbf{S}, N)$ satisfies the distribution laws, then $\mathbf{T}=\mathbf{T}_{\min , \min }$ and $\mathbf{S}=\mathbf{S}_{\max , \max }$, but then $\mathbf{T}$ does not satisfy the law of contradiction, since for any $\mathbf{x} \in L([0,1]), N(\mathbf{x}) \in L([0,1])$ (since $N$ is involutive), so $\mathbf{T}(\mathbf{x}, N(\mathbf{x})) \geq_{L} 0_{L}$.

Theorem 10. Let $(\mathbf{T}, \mathbf{S}, N)$ be a dual triple that satisfies the law of the excluded middle and the law of contradiction. Then $(\mathbf{T}, \mathbf{S}, N)$ does not satisfy the distributive laws.

\subsection{Interval-Valued Fuzzy Implication Operators}

In fuzzy set theory, a fuzzy implication operator $I$ is a function $[0,1]^{2} \rightarrow[0,1]$ that fulfills a certain set of properties (see [20]), so that $I\left(\mu_{A}(x), \mu_{B}(y)\right)$ represents the degree of truth of the fuzzy conditional If $x$ is $A$ then $y$ is $B$, where $A$ and $B$ are fuzzy sets. 
A possible adaptation of the concept of the fuzzy implication operator to the case of IVFS theory has been given in [62] in the following way.

Definition 6. An interval-valued fuzzy implication operator is a function

$$
I_{I V}:(L([0,1]))^{2} \rightarrow L([0,1])
$$

that has the following properties:

$I_{I V 0}$. If $\mathbf{x}, \mathbf{y} \in L([0,1])$ are such that $K_{0}(\mathbf{x})=K_{1}(\mathbf{x})$ and $K_{0}(\mathbf{y})=K_{1}(\mathbf{y})$, then $W_{I_{I V}(\mathbf{x}, \mathbf{y})}=0$.

$I_{I V 1}$. If $\mathbf{x} \leq_{L} \mathbf{x}^{\prime}$, then $I_{I V}(\mathbf{x}, \mathbf{y}) \geq_{L} I_{I V}\left(\mathbf{x}^{\prime}, \mathbf{y}\right)$ for all $\mathbf{y} \in L([0,1])$.

$I_{I V 2}$. If $\mathbf{y} \leq_{L} \mathbf{y}^{\prime}$, then $I_{I V}(\mathbf{x}, \mathbf{y}) \leq_{L} I_{I V}\left(\mathbf{x}, \mathbf{y}^{\prime}\right)$ for all $\mathbf{x} \in L([0,1])$.

$I_{I V 3} . I_{I V}\left(0_{L}, \mathbf{x}\right)=1_{L}$ for all $\mathbf{x} \in L([0,1])$.

$I_{I V 4} . I_{I V}\left(\mathbf{x}, 1_{L}\right)=1_{L}$ for all $\mathbf{x} \in L([0,1])$.

$I_{I V 5} . I_{I V}\left(1_{L}, 0_{L}\right)=0_{L}$.

If $\mathbf{x}, \mathbf{y} \in L([0,1]), I_{I V 0}$ establishes that if the sets are fuzzy, that is, the length of the intervals is zero, then the length of the interval-valued fuzzy implication operator is zero. Moreover, if in Definition 6 we replace $L([0,1])$ by $[0,1]$ and we eliminate the condition $I_{I V 0}$, then we have the definition of a fuzzy implication operator in the sense of Fodor (see [20]).

This definition (Definition 6) extends classical two-valued implication; that is

$$
I_{I V}\left(0_{L}, 0_{L}\right)=I_{I V}\left(0_{L}, 1_{L}\right)=I_{I V}\left(1_{L}, 1_{L}\right)=1_{L} \quad \text { and } \quad I_{I V}\left(1_{L}, 0_{L}\right)=0_{L} .
$$

In [73], Cornelis et al. presented the following definition: An implicator on $L([0,1])$ is any $(L([0,1]))^{2} \rightarrow L([0,1])$ mapping $\mathcal{I}$ satisfying $\mathcal{I}\left(0_{L}, 0_{L}\right)=\mathcal{I}\left(0_{L}, 1_{L}\right)=\mathcal{I}\left(1_{L}, 1_{L}\right)=1_{L}$ and $\mathcal{I}\left(1_{L}, 0_{L}\right)=0_{L}$. Moreover, we require $\mathcal{I}$ to be decreasing in its first component and increasing in its second component.

Beginning from this concept, $S$-implicators on $L([0,1])$ and $R$-implicators on $L([0,1])$ were defined in [73]. The axioms of Smets and Magrez (see [87]) were adapted to the case of IVFSs, and various characterization theorems for $S$ - and $R$-implicators on $L([0,1])$ were analyzed. It was also made clear that every interval-valued implication operator $I_{I V}$ (Definition 6) is an implicator on $L([0,1])$.

We must point out that the characterization and construction theorems for the implicators on $L([0,1])$ (see [73]) are a generalization of the construction and characterization theorems for fuzzy $S$-implications and fuzzy $R$-implications (see [20]). In this connection, we see the following open problem. In the case of fuzzy sets, various characterizations of fuzzy $S$-implications, fuzzy $R$-implications, fuzzy $Q L$ implications, and fuzzy $D$-implications have been studied, using in some cases the property $I(x, n(x))=$ $n(x)$ for all $x \in[0,1]$. It is now necessary to carry out a study parallel to that in $[88,89]$, adapting the definitions and properties described in those publications to the implicators on $L([0,1])$ defined above.

In [62], the properties usually required of interval-valued fuzzy implication operators are presented. These properties can be divided into two groups: the ones that result from adapting the properties of fuzzy implication operators to the interval-valued case and those that are interval-valued per se. For the latter group, the following three properties have been proposed, among others:

$I_{I V 6} . W_{I_{I V}(\mathbf{x}, \mathbf{y})} \leq \vee\left(1-K_{0}(\mathbf{x}), 1-K_{1}(\mathbf{x}), 1-K_{0}(\mathbf{y}), 1-K_{1}(\mathbf{y})\right)$.

$I_{I V 7}$. If $\mathbf{x}=\mathbf{y}$, then $W_{I_{I V}}(\mathbf{x}, \mathbf{y})=W_{\mathbf{x}}$.

$I_{I V 8}$. If $W_{\mathbf{x}}=W_{\mathbf{y}}$, then $W_{I_{I V}(\mathbf{x}, \mathbf{y})}=W_{\mathbf{x}}$.

Note that if the property $I_{I V 8}$ holds, then $I_{I V 7}$ holds, but the converse does not hold. Evidently, neither $I_{I V 7}$ nor $I_{I V 8}$ is in contradiction with $I_{I V 6}$.

\subsubsection{A Construction Method}

In [73], various methods for the construction of implicators on $L([0,1])$ are presented. These methods are characterized by the fact that they always use the extremes of the intervals. However, in the construction method for interval-valued fuzzy implication operators that we shall present next (see [62]), other points 
not obtained by applying $t$-norms and $t$-conorms in $[0,1]$ to the extremes of the intervals, such as the average point of the interval, can also be used. This method comes out of the following interpretation of the condition rule (with IVFSs).

In the theory of IVFSs, a general rule for an expert system has the form If $u$ is $A$ then $v$ is $B$, where $u$ is a variable taking values in $U, v$ is a variable taking values in $V, A \in \mathcal{I} V F S s(U)$, and $B \in \mathcal{I} V F S s(V)$.

The interval $A(u)$ is the truth degree of the proposition ' $u$ is $A$.' Let us take two values, the extremes of the intervals, $K_{0}(A(u))$ and $K_{1}(A(u))$. Since in reality we are interested in assigning a single value to the degree of truth of the proposition ' $u$ is $A$,' we shall say that it is given by an aggregation of $K_{0}(A(u))$ and $K_{1}(A(u))$, that is, by $M_{1}\left(K_{0}(A(u)), K_{1}(A(u))\right)$. The choice of the aggregation will depend on the experimental situation we are dealing with.

With respect to ' $v$ is $B$,' we can say the same. As before, we shall say that the truth degree of the proposition ' $v$ is $B$ ' is given by an aggregation of $K_{0}(B(v))$ and $K_{1}(B(v))$, that is, $M_{2}\left(K_{0}(B(v)), K_{1}(B(v))\right)$.

Therefore, bearing in mind these considerations and the interpretation of the fuzzy implication operator $I$, we shall say that a value that represents the truth degree of the interval-valued fuzzy conditional If $u$ is $A$ then $v$ is $B$ (where $A, B$ are interval-valued fuzzy sets) is given by

$$
I\left(M_{1}\left(K_{0}(A(u)), K_{1}(A(u))\right), M_{2}\left(K_{0}(B(v)), K_{1}(B(v))\right)\right),
$$

$I$ being any fuzzy implication operator in Fodor's sense.

We can perform an analogous reasoning for the degree of non-truth of the proposition: if we take as the non-truth degree of the proposition ' $u$ is $A$ ' two values that are the negation of the extremes of the intervals, we obtain the result that the degree of non-truth of the proposition ' $u$ is $A$ ' will be given by any aggregation of $1-K_{1}(A(u))$ and $1-K_{0}(A(u))$, that is, by $M_{3}\left(1-K_{1}(A(u)), 1-K_{0}(A(u))\right)$. Likewise, the non-truth degree of the proposition ' $v$ is $B$ ' will be given by $M_{4}\left(1-K_{1}(B(v)), 1-K_{0}(B(v))\right), M_{4}$ being an aggregation operator.

Following further analogous reasoning, we have the result that the non-truth degree of the intervalvalued fuzzy conditional If $u$ is $A$ then $v$ is $B$ is given by

$$
\left.1-I^{\prime}\left(1-M_{3}\left(1-K_{1}(A(u)), 1-K_{0}(A(u))\right)\right), 1-M_{4}\left(1-K_{1}(B(v)), 1-K_{0}(B(v))\right)\right),
$$

$I^{\prime}$ being any fuzzy implication operator in Fodor's sense.

Evidently, we can say that another value represents the degree of truth of the interval-valued fuzzy conditional If $u$ is $A$ then $v$ is $B$ (where $A$ and $B$ are interval-valued fuzzy sets). This is given by

$$
\left.I^{\prime}\left(1-M_{3}\left(1-K_{1}(A(u)), 1-K_{0}(A(u))\right)\right), 1-M_{4}\left(1-K_{1}(B(v)), 1-K_{0}(B(v))\right)\right) .
$$

Therefore, we can interpret the interval

$$
\begin{aligned}
& {\left[I\left(M_{1}\left(K_{0}(A(u)), K_{1}(A(u))\right), M_{2}\left(K_{0}(B(v)), K_{1}(B(v))\right)\right),\right.} \\
& \left.\left.\quad I^{\prime}\left(1-M_{3}\left(1-K_{1}(A(u)), 1-K_{0}(A(u))\right)\right), 1-M_{4}\left(1-K_{1}(B(v)), 1-K_{0}(B(v))\right)\right)\right]
\end{aligned}
$$

as the truth degree of the interval-valued fuzzy conditional If $u$ is $A$ then $v$ is $B$. (Obviously, in the expression above, $I, I^{\prime}, M_{1}$, etc., are taken such that the expression is an interval.)

In the following proposition, we present a construction method for interval-valued fuzzy implication operators in the sense of Definition 6 (see [62]). The aggregations that we use in this construction method are the following: an aggregation operator is a $[0,1]^{2} \rightarrow[0,1]$ mapping $M$ that satisfies the following conditions:

1. $M(0,0)=0 ; M(1,1)=1$.

2. $M$ is increasing in its first and second argument.

3. $M(x, y)=M(y, x)$ for all $x, y \in[0,1]$. 


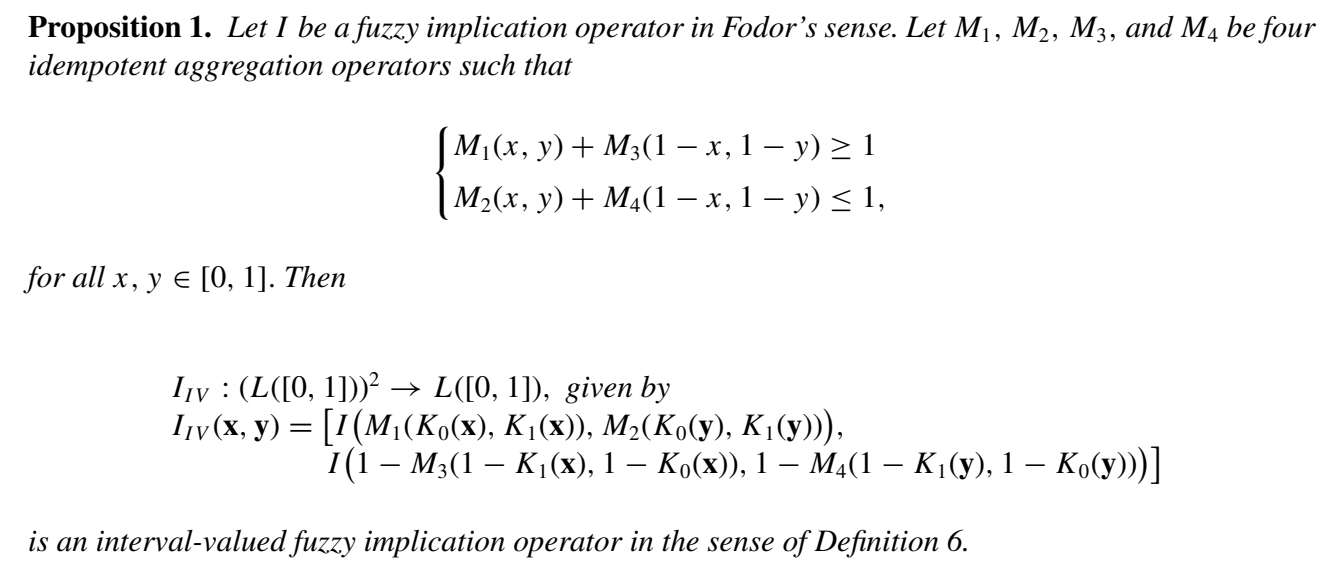

$$
\left\{\begin{array}{l}
M_{1}(x, y)+M_{3}(1-x, 1-y) \geq 1 \\
M_{2}(x, y)+M_{4}(1-x, 1-y) \leq 1
\end{array}\right.
$$

for all $x, y \in[0,1]$. Then

$$
\begin{aligned}
& I_{I V}:(L([0,1]))^{2} \rightarrow L([0,1]), \text { given by } \\
& \begin{aligned}
I_{I V}(\mathbf{x}, \mathbf{y})= & {\left[I\left(M_{1}\left(K_{0}(\mathbf{x}), K_{1}(\mathbf{x})\right), M_{2}\left(K_{0}(\mathbf{y}), K_{1}(\mathbf{y})\right)\right),\right.} \\
& \left.I\left(1-M_{3}\left(1-K_{1}(\mathbf{x}), 1-K_{0}(\mathbf{x})\right), 1-M_{4}\left(1-K_{1}(\mathbf{y}), 1-K_{0}(\mathbf{y})\right)\right)\right]
\end{aligned}
\end{aligned}
$$

is an interval-valued fuzzy implication operator in the sense of Definition 6.

In [62], there is a study of the conditions under which the constructions of Proposition 1 fulfill the properties $I_{I V 6}-I_{I V 8}$, among others.

Example 1. If $M_{1}=M_{3}=\max$ and $M_{2}=M_{4}=\min$, then we obtain the interval-valued fuzzy implication operator introduced by Jenei (see [30]); that is,

$$
I_{I V}(\mathbf{x}, \mathbf{y})=\left[I\left(K_{1}(\mathbf{x}), K_{0}(\mathbf{y})\right), I\left(K_{0}(\mathbf{x}), K_{1}(\mathbf{y})\right)\right]
$$

Example 2. If, under the conditions of Example 1, we take the Kleene-Dienes fuzzy implication operator, that is, $I(x, y)=\max (1-x, y)$, we have the expression for the first interval-valued fuzzy operator introduced by Atanassov ( see [35]),

$$
I_{I V}(\mathbf{x}, \mathbf{y})=\left[\max \left(1-K_{1}(\mathbf{x}), K_{0}(\mathbf{y})\right), \max \left(1-K_{0}(\mathbf{x}), K_{1}(\mathbf{y})\right)\right]
$$

In [62], it is proved that the expression in Example 2 satisfies the properties $I_{I V 6}, I_{I V 7}$, and $I_{I V 8}$. Cornelis et al. proved in [73] that this expression is an $S$-implicator on $L([0,1])$. Moreover, they also proved that if, under the conditions of Proposition 1, we take $M_{1}(x, y)=M_{2}(x, y)=M_{3}(x, y)=M_{4}(x, y)=(x+$ $y) / 2$ and the Kleene-Dienes implication operator, the expression that we obtain is not an $S$-implicator on $L([0,1])$ or an $R$-implicator on $L([0,1])$.

In [73], the definition of an interval-valued fuzzy $S$-implication (in Fodor's sense) is presented and in [63] the conditions under which these implications satisfy property $I_{I V 7}$ are studied. In this connection, the following open problems are worth mentioning:

1. To study the cases for which interval-valued fuzzy S-implications satisfy the property $I_{I V 8}$.

2. To analyze the conditions that must be met in order for interval-valued fuzzy R-implications to satisfy the property $I_{I V 8}$.

3. To define interval-valued fuzzy D-implications (see [88]) and study the conditions under which these implications satisfy $I_{I V 8}$ and the property that results from generalizing the fuzzy property $I(x, n(x))=$ $n(x)$ for all $x \in[0,1]$ to the case of IVFSs.

\subsection{Information Measures}

The purpose of this section is to introduce the main developments and results regarding the best-known interval-valued information measures. The study that we present is for finite referentials. The non-finite case has been analyzed in the corresponding references. 


\section{Indetermination Index}

In 1975, Sambuc [4] presented the following definition: given $A \in \operatorname{IVFSs}(U)$, the indetermination index of the set $A$ is the following expression:

$$
\mathcal{J}(A)=\frac{\sum_{i=1}^{\mathcal{N}} W\left(A\left(u_{i}\right)\right)}{\mathcal{N}}, \quad \text { where } \mathcal{N}=\operatorname{Card}(U)
$$

Sambuc used (2) to determine how far the IVFS considered was from the corresponding fuzzy set that he would have taken if he had used fuzzy set theory in his work. In fact, he reached the expression (2) by applying the concept of the Hamming distance between the extremes of the intervals. He also presented an expression for this index when the Euclidean distance was used for its construction.

\section{Distances}

A lot has been written on the concept of distance between IVFSs. In the following definition, we present the currently most commonly used expressions (see [90, 91]).

Definition 7. Let $U(\operatorname{Card}(U)=\mathcal{N})$ be the referential set. We define the following distances:

1. The normalized Euclidean distance between A, B belonging to $\mathcal{I V F S s}(U)$,

$$
D_{W}^{E}(A, B)=\sqrt{\frac{1}{\mathcal{N}} \sum_{i=1}^{\mathcal{N}} \frac{\left(K_{0}\left(A\left(u_{i}\right)\right)-K_{0}\left(B\left(u_{i}\right)\right)\right)^{2}+\left(K_{1}\left(A\left(u_{i}\right)\right)-K_{1}\left(B\left(u_{i}\right)\right)\right)^{2}+\left(W\left(A\left(u_{i}\right)\right)-W\left(B\left(u_{i}\right)\right)\right)^{2}}{2}} .
$$

2. The normalized Hamming distance between $A, B \in \mathcal{I} V F S s(U)$,

$D_{W}^{H}(A, B)=\frac{1}{\mathcal{N}} \sum_{i=1}^{\mathcal{N}} \frac{\left|K_{0}\left(A\left(u_{i}\right)\right)-K_{0}\left(B\left(u_{i}\right)\right)\right|+\left|K_{1}\left(A\left(u_{i}\right)\right)-K_{1}\left(B\left(u_{i}\right)\right)\right|+\left|W\left(A\left(u_{i}\right)\right)-W\left(B\left(u_{i}\right)\right)\right|}{2}$.

3. The normalized Hausdorff distance between $A, B \in \mathcal{I V F S}(U)$,

$$
\begin{gathered}
\mathcal{D}_{W}(A, B)=\frac{1}{\mathcal{N}} \sum_{i=1}^{\mathcal{N}} \max \left(\left|K_{0}\left(A\left(u_{i}\right)\right)-K_{0}\left(B\left(u_{i}\right)\right)\right|,\left|K_{1}\left(A\left(u_{i}\right)\right)-K_{1}\left(B\left(u_{i}\right)\right)\right|,\right. \\
\left.\left|W\left(A\left(u_{i}\right)\right)-W\left(B\left(u_{i}\right)\right)\right|\right) .
\end{gathered}
$$

Historically, the expressions developed in Definition 7 were defined without including the term relative to the length of the intervals (see $[4,34,60,61]$ ).

\section{Degree of Compatibility}

In 1987, Gorzalczany (see [9]) defined the degree of compatibility between two interval-valued fuzzy sets $A$ and $B$ on the same referential $U$ in the following way:

The degree of compatibility $\Gamma(A, B)$ of an interval-valued fuzzy set $A$ (such that there is at least one $u \in U$ with $\left.K_{0}(u) \neq 0\right)$ with an interval-valued fuzzy set $B$ being an element of $L([0,1])$ is given by

$$
\begin{aligned}
\Gamma(A, B)= & {\left[\min \left(\frac{\max _{u \in U}\left(\min \left(K_{0}(A(u)), K_{0}(B(u))\right)\right)}{\max _{u \in U} K_{0}(A(u))}, \frac{\max _{u \in U}\left(\min \left(K_{1}(A(u)), K_{1}(B(u))\right)\right)}{\max _{u \in U} K_{1}(A(u))}\right),\right.} \\
& \left.\max \left(\frac{\max _{u \in U}\left(\min \left(K_{0}(A(u)), K_{0}(B(u))\right)\right)}{\max _{u \in U} K_{0}(A(u))}, \frac{\max _{u \in U}\left(\min \left(K_{1}(A(u)), K_{1}(B(u))\right)\right)}{\max _{u \in U} K_{1}(A(u))}\right)\right] .
\end{aligned}
$$

A study of the properties of this concept and a very interesting application of it can be found in [60].

\section{Entropies}

There are two different definitions in the literature of the concept of an interval-valued fuzzy entropy (IV entropy). The first, $\mathcal{E}_{F}$, was presented in 1996 in [61], and the second, $\mathcal{E}_{c}$, in 2001 in [91]. The difference between the two definitions lies in the fact that $\mathcal{E}_{F}$ is a measure of how far an IVFS is from a 
fuzzy set, whereas $\mathcal{E}_{c}$ is a measure of how far an IVFS is from a crisp set. Therefore, $\mathcal{E}_{F}$ is based on the ideas of Sambuc and $\mathcal{E}_{c}$ is based on the concept of fuzzy entropy.

Definition 8. A real function $\mathcal{E}_{F}: \mathcal{I V F S s}(U) \rightarrow \mathbb{R}^{+}$is called an entropy on $\mathcal{I V F S s}(U)$ if $\mathcal{E}_{F}$ has the following properties:

(IF1) $\mathcal{E}_{F}(A)=0$ if and onlu if $A \in \mathcal{F} S s(U)$.

$(I F 2) \mathcal{E}_{F}(A)=\operatorname{Card}(U)=\overline{\overline{\text { f }}}$ and only if $K_{0}(A(u))=0$ and $K_{1}(A(u))=1$ for all $u \in U$.

(IF3) $\mathcal{E}_{F}(A)=\mathcal{E}_{F}\left(A_{N}\right)$ for all $A \in \mathcal{I V F S s}(U)$. ( $N$ is the involutive and strict $I V$ negation generated by

$n(x)=1-x$ for all $x \in[0,1]$ according to Theorem 2.)

(IF4) If $A \preceq B$, then $\mathcal{E}_{F}(A) \geq \mathcal{E}_{F}(B)$.

In [61], these entropies $\mathcal{E}_{F}$ are studied in depth and a theorem for their construction from functions $\varphi:[0,1] \rightarrow[0,1]$ is presented. It is also said there that the most commonly used expression is the one obtained when we take $\varphi(x)=x$ so that $\mathcal{E}_{F}(A)=\mathcal{J}(A)$ for all $A \in \mathcal{I} V F S s(U)$. The edge detector developed in [54] for objects in an image uses the IV entropy $\mathcal{E}_{F}$.

Definition 9. A real function $\mathcal{E}_{c}: \mathcal{I V F S s}(U) \rightarrow \mathbb{R}^{+}$is called an entropy on $\mathcal{I} V F S s(U)$ if $\mathcal{E}_{c}$ has the following properties:

(Ic1) $\mathcal{E}_{c}(A)=0$ if and only if $A$ is a crisp set.

(Ic2) $\mathcal{E}_{c}(A)=1$ if and only if $K_{0}(A(u))=1-K_{1}(A(u))$ for all $u \in U$.

$(I c 3) \mathcal{E}_{c}(A)=\mathcal{E}_{c}\left(A_{N}\right)$ for all $A \in \mathcal{I} V F S s(U)$. ( $N$ is the involutive and strict IV negation generated by $n(x)=1-x$ for all $x \in[0,1]$ according to Theorem 2 .)

(Ic4) $\mathcal{E}_{c}(A) \leq \mathcal{E}_{c}(B)$ if $K_{0}(A(u)) \leq K_{0}(B(u))$ and $K_{1}(A(u)) \leq K_{1}(B(u))$ for $K_{0}(B(u)) \leq 1-K_{1}(B(u))$

or $K_{0}(A(u)) \geq K_{0}(B(u))$ and $K_{1}(A(u)) \geq K_{1}(B(u))$ for $K_{0}(B(u)) \geq 1-K_{1}(B(u))$.

A study of the main properties of $\mathcal{E}_{c}$ can be found in [39,91]. In this latter paper, a first attempt to relate $\mathcal{E}_{F}$ to $\mathcal{E}_{c}$ by means of a novel construction method is presented.

The idea of relating $\mathcal{E}_{c}$ to IV similarities using item (2) of Definition 7 has led Szmidt and Kacprzyk to modify the definition of $\mathcal{E}_{c}$ (see [92]).

It is necessary to point out that Tizhoosh (see [50]) experimentally proves that in the determination of the threshold of an image, the entropy $\mathcal{E}_{F}$ provides very good results. This author at no time considers the IV entropy: $\mathcal{E}_{c}$.

In spite of the results obtained by Tizhoosh, we consider that we have the following open problem: defining interval-valued fuzzy entropy so that it gives as a result an element of $L([0,1])$ and not an element of $[0,1]$. We should also study the conditions under which we recover Definitions 8 and 9 from the new definition and in this way we can analyze the reasons why the use of $\mathcal{E}_{F}$ gives good results in threshold computation in image processing.

\section{Similarity}

In [60], there is also a section on the similarity of IVFSs. normal interval-valued similarity measure $S(A, B)$ between two IVFSs $A$ and $B$ is defined as one that satisfies the following five properties: (i) $S(A, B)=S(B, A)$ for all $A, B \in \mathcal{I V F S s}(U)$; (ii) $S\left(D, D_{C}\right)=0_{L}$ for all $D \in \mathcal{P}(U)$, where $D_{C}$ is the complement of $D$ and $\mathcal{P}(U)$ is the class of all crisp sets of $U$; (iii) $S(C, C)=1_{L}$ for all $C \in \mathcal{I} V F S s(U)$; (iv) for all $A, B, C \in \mathcal{I V F S s}(U)$, if $A \leq B \leq C$, then $S(A, B) \geq S(A, C)$ and $S(B, C) \geq S(A, C)$; and (v) if $A, B \in \mathcal{I} V F S s(U)$, then $S(A, B) \in L([0,1])$. In [60], it is proved that the relation

$$
S(A, B)=\left[S_{L}(A, B), S_{U}(A, B)\right],
$$

where $S_{L}(A, B)$ is a fuzzy similarity measure (see [93]) between the lower membership functions of $A$ and $B$, and $S_{U}(A, B)$ is a fuzzy similarity measure between the upper membership functions of $A$ 
and $B$, satisfies (i)-(v). There are additional results, but these are beyond the scope of this chapter.

Starting from the work of Mitchell (see [94]), Mendel presented in [17] a new expression for IV similarities. In the same paper, Mendel posed the following open question: finding the possible connection (if there is one) between the similarity measure (3) and the expression that he proposes.

The IV similarities defined in $[17,60]$ have in common the fact that they give an interval as a result, that is, an element of $L([0,1])$. However, the IV similarities proposed in [70, 95-98] are an adaptation of the well-known measures of similarity between fuzzy sets, so that they give an element of $[0,1]$ as a result. Evidently, in this case the expressions that relate IV entropy $\left(\mathcal{E}_{c}\right)$, IV similarity, and IV distances are identical to those obtained by Liu (see [93]) in 1992 for fuzzy sets. At this point, we would like to ask the following question:

\section{Should the information measures between IVFSs be such that they give as a result an element of} $L([0,1])$ or an element of $[0,1]$ ?

We think that this is the first problem that we should approach. Our particular opinion is that these measures should give elements of $L([0,1])$ as a result. Furthermore, we think that if we have an intervalvalued measure $\Pi$ (e.g., the IV similarity), such that for each pair of elements $A, B \in \mathcal{I V F S s}(U)$ it gives as the result the interval $\Pi(A, B) \in L([0,1])$, then it should turn out that for certain values of $\alpha \in[0,1]$ when a fuzzy measure $\pi$ is applied (e.g., fuzzy similarity) in association with $\Pi$, the following equality holds:

$$
K_{\alpha}(\Pi(A, B))=\pi\left(K_{\alpha}(A), K_{\alpha}(B)\right) .
$$

We consider that in the future we must analyze the conditions under which information measures satisfy (4). Evidently, these measures should be defined so that they give as a result an element of $L([0,1])$.

We have said before that in the future we must define the entropy of interval-valued fuzzy sets as an element of $L([0,1])$. Once this definition has been made we must analyze different methods for the construction of these entropies and study their generation from IV similarities. We must also relate IV distances, IV similarities, and the new definition of IV entropy. To do this we must bear in mind each and every one of the ideas described in the references used up to now on the topic and on the results obtained in the following works: $[75,100-106]$.

\section{Inclusion Measures, IV Correlation, and IV Information Energy}

In [60], inclusion measures between IVFSs were studied for the first time. These measures give an element of $L([0,1])$ as a result. Also, various methods of construction of these measures from fuzzy implication operators were analyzed. In 2003, Kehagias and Konstantinidou proposed a new version of these measures [107].

In [75], Cornelis and Kerre present a definiti $\equiv$ interval-valued inclusion measure in the same sense as the one presented in [60]; that is, the measure an axiomatization that enables them to relate their inclusion measure with the expression of IV entropy $\mathcal{E}_{c}$. We consider that any study done to relate IV entropy, IV similarity, and IV distance must take into account the results obtained by these authors in order to generate $\mathcal{E}_{c}$ from IV inclusion measures. We must also highlight the theoretical development made for using IV inclusion measures in approximate re $\equiv$ ing.

ॠraly, a method for the construction of interval-valued fuzzy entropies from IV inclusion measures is presented in [96]. However, in this case the method proposed is an adaptation to IVFSs of well-known results of fuzzy set theory, for the authors believe that the result of any information measure with IVFSs should always be an element of $[0,1]$.

Gerstenkorn and Manko [108] introduced the concepts of IV correlation and IV information energy. Later, in [109], a detailed study of these concepts was carried out. 


\subsection{Some Applications}

Our goal in these pages is not to go into all the details of each and every one of the fields where IVFSs are being applied; we wish to present only some representative contributions. Nevertheless, we think that we should make the following remarks:

1. In most of the applications that we are going to present it is proved that when we have great imprecision in the determination of the membership degrees, better results are obtained by modelizing with IVFSs than with FSs.

2. The use of IVFSs does not increase the complexity of the algorithms, it only increases the number of necessary calculations for each algorithm. On the other hand, bearing in mind the latest technological advances, it results that the expense in time for the execution of the algorithms with IVFSs is practically the same as that of the algorithms with FSs. (This fact is made especially clear in algorithms that use IVFSs for image processing.)

\section{Approximate Reasoning}

Approximate reasoning is, formally speaking, as Turksen says [110], the process or processes by which a possible imprecise conclusion is deduced from a collection of imprecise premises. In this section, we present a short review of the inference methods most commonly used in the literature when the imprecise premises and the imprecise conclusions are represented using IVFSs.

The generalized modus ponens (GMP) inference rule with IVFSs is represented in the following way (see $[9,65,73,111])$ :

$$
\begin{gathered}
\text { If } u \text { is } A \text { then } v \text { is } B \\
u \text { is } A^{\prime} \\
v \text { is } B^{\prime},
\end{gathered}
$$

where $u$ is a variable taking values in $U, v$ is a variable taking values in $V, A, A^{\prime} \in \mathcal{I} V F S s(U)$, and $B, B^{\prime} \in \mathcal{I V F S s}(V)$.

The methods given in order to obtain the conclusion $B^{\prime}$ can be divided into two groups: the ones that use an adaptation of Zadeh's compositional rule (see $[1,112])$ to the interval-valued fuzzy case and those that do not.

The idea of applying Zadeh's compositional rule to the GMP with IVFSs led to the study of intervalvalued fuzzy relations (IVFRs). In [64, 65, 67, 80, 113], the properties of IVFRs were analyzed and the composition of such relations was studied. Afterward, these relations were applied to the computation of the conclusion of the GMP with IVFSs. In [114], interval-valued fuzzy equations were studied for the first time (see [115]). We must say that the field of IVFRs is the least studied of those presented in this chapter.

Arnould and Tano in 1995 (see [116]) constructed an expert system using rules with IVFSs. In the inference engine of that expert system, Zadeh's compositional rule is first applied to the lower extremes of the intervals and then to the upper extremes.

With respect to the second group, that is, the applications that do not use Zadeh's composition rule, it is worth pointing out that all of them use the algorithm proposed by Gorzalczany (see [12]). This algorithm consists of two steps:

1. Relate $A$ to $A^{\prime}$ by means of an information measure.

2. Build the consequence $B^{\prime}$ using the result of the comparison above and $B$.

In [12], Gorzalczany used for step 1 the concept of the degree of compatibility, whereas in [60] first the degree of inclusion was used and then the IV similarities. Note that the measures used always give an element of $L([0,1])$ as a result.

We must point out that regardless of the method used for calculating $B^{\prime} \in \mathcal{I} V F S s(V)$, we must always study properties of the type 'if $A^{\prime}=A$, then $B^{\prime}=B$,' etc. In this connection, in [60] there is an in-depth 
analysis of the conditions under which the methods developed in that paper satisfy the axioms of Fukami et al. (see [117]) or the axioms of Baldwin and Pilsworth (see [118]). A similar study has been carried out in $[65,73]$ for the methods that use Zadeh's compositional rule adapted to the interval-valued fuzzy case.

In $[12,64,65,68,70,116,119]$, various methods for obtaining the conclusion from a system of interval-valued rules, in addition to the GMP with IVFSs, can be found.

\section{Image Processing}

A very important problem in image processing is the detection of the edges of the objects that make up an image. A pixel is said to belong to an edge if it has associated with it a big enough change in intensity. In [54], each image is associated with an IVFS so that the length of the interval associated with each pixel represents the intensity change between that pixel and its neighbors. Therefore, if the length of the interval is large enough, then the pixel belongs to an edge. The edge detector developed in [54] surpassed the classical detectors in the literature in three of the four types in which images were classified. In [54], an expression for calculating the contrast of an image using interval-valued $S$-implication operators is given.

In Section 22.2.2 we have said that Tizhoosh developed a method for calculating the threshold of an image using IVFSs. In [50] and later in [51] and [52] it has been experimentally proved that when working with images that have a large number of pixels such that the experts are not able to determine precisely whether they belong to the background or to the object, this method provides better results than the rest of known methods. In any other type of images, it has also been proved that the results obtained with this algorithm are similar to those obtained with algorithms that do not use IVFSs.

The algorithm proposed by Tizhoosh for images with $L$ levels of gray consists of the following steps:

1. Assign $L$ fuzzy sets to each image $Q$.

2. Associate with each fuzzy set its corresponding IVFS constructed with the method described in Section 22.2.2.

3. For each IVFS, calculate $\mathcal{E}_{F}$.

4. Take as threshold the intensity corresponding to IVFS with lowest value of $\mathcal{E}_{F}$.

We must point out that Tizhoosh calls $\mathcal{E}_{F}$ (see again [61]) ultrafuzziness and that the algorithm above has been generalized in [51, 52]. In Figure 22.2 we give an example where it is proved that we obtain the best result with this algorithm. In [54] it has been proved that the time and memory efficiency of the algorithms that use IVFSs (for image processing) is practically the same as the efficiency of those that do not use IVFSs.

In [120], Gaussian noise was eliminated from an image using algorithms with IVFSs. In general, each and every one of the applications constructed with IVFSs for image processing (when it is applied to images with great imprecision in the membership of the pixels that compose it) gives better results than those constructed with fuzzy sets. This is due to the fact that they use a characteristic of IVFSs that fuzzy sets do not have, namely, length (of interval).

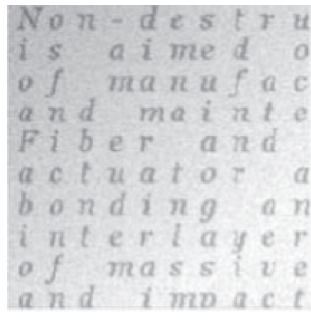

(a)

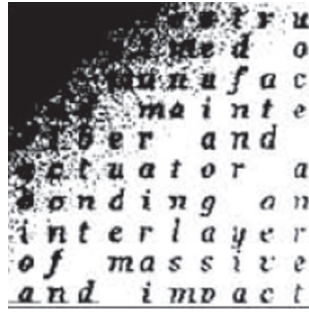

(b)

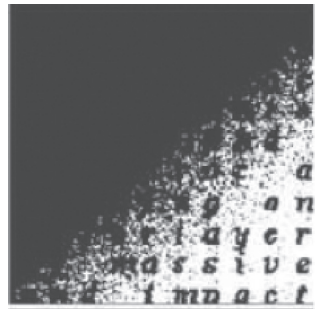

(c)

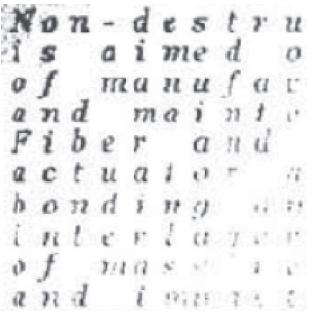

(d)

Figure 22.2 (a) Original image; (b) image binarized with the classical Otsu method; (c) image binarized with the Huang fuzzy method; (d) image binarized with IVFS method 


\section{Computing with Words \\ In [121] Zadeh defines computing with words (CWW) in the following way: a theory in which the objects of computation are words and propositions drawn from a natural language. We know that words can have different meanings depending on the person and the context in which the person is. Therefore there is uncertainty associated with the words (see [17, 59, 122]). This reasoning has made several authors consider it necessary to use IVFSs in CWW (see [123]).}

\section{Decision Making}

In decision making, one works with many types of information, among them interval-valued information. In [8], various methods of processing information represented in this way are analyzed. Furthermore, in $[124,125]$, there is a study of several processes of aggregation of non-homogeneous information with contexts composed of numerical, interval-valued, and linguistic values. It is also worth pointing out the work of Bilgiç (see, e.g., [67]). More applications in decision making can be found in [126-128].

\section{Other Applications}

Obviously there exist a lot of applications of IVFSs; here we present the most representative. For applications to fuzzy linear programming, see $[69,129]$. For economics, see [130, 131]. For medicine, see $[4,113]$. For robotics, see [118, 132-134]. For fuzzy modeling, see [135-137]. For Web intelligence, see [56]. For the theory of possibility, see [73]. For control, see [10, 57].

\subsection{Granular Computing and Interval-Valued Fuzzy Sets}

The topic of fuzzy information granulation was first proposed and discussed by Zadeh in 1979 (see [138]). In this paper, a granule was defined as a collection of indistinguishable objects and granular information as the grouping of objects into granules. Later, Zadeh established in [139] the following: the theory of fuzzy information granulation is inspired by the ways in which humans granulate information and reason with it. On the basis of these considerations Yao (see [140]) says that granular computing may be regarded as a label of theories, methodologies, techniques, and tools that make of granules, that is, groups, classes, or clusters of a universe, in the process of problem solving.

Therefore, granular computing is based on the principle that the description of the world around us by means of techniques that use exclusively numeric precision is often unnecessary and has a very high cost. Furthermore, granular computing also considers the fact that human thinking does not work at a numeric level of precision but at a much more abstract level. In this sense in [81] the following is established: granular computing is a formalism for expressing that abstraction within computational processes, thus endowing computer systems with a more human-centric view of the world. The central notion in granular computing is that there are many 'levels' of precision in which information about the real world can be expressed, with numeric precision being the most refined and a binary value the coarsest (see [141]).

A granule can be interpreted as one of the numerous particles or elements that compose a unit. The size of the granule is its basic property. Intuitively the size can be interpreted as the degree of abstraction, detail, or precision.

In many applications, when the problem to solve deals with imprecise or vague information, it can be difficult to identify specific data and then we are forced to use granules. Furthermore, we have said in the introduction that the use of IVFSs arises in the applications where it is hard to precisely determine the value of the membership function for the elements. These two arguments allow us to say that IVFSs can be used in the tasks of representing, operating, and reasoning with granules (see [138]).

Depending on the application, a granule can be an element, that is, an interval, or it can be represented by an IVFS. In Section 22.2.2 we have seen that we can construct IVFSs from fuzzy sets; therefore, we can construct hierarchies of granules and represent arbitrary groups (clusters) of granules using IVFSs. This representation has the advantage that it enables us to use all of the results known for IVFSs in granular computing. 


\subsection{Conclusion}

In this chapter, we have reviewed the basic concepts of IVFSs. We have focused on the elementary properties and have given a detailed set of references for more advanced properties. We have also posed several unsolved problems and have demonstrated the importance that the study of properties that differentiate them from fuzzy sets should have in future research.

As with all young theories, there are a great number of open problems in IVFS theory. We have presented some of them at various points in this chapter.

We have detected that currently there is general interest in finding applications of type 2 fuzzy sets and that the best results are almost always obtained when IVFSs are used. For this reason, we believe that these sets are going to be thoroughly studied and used in the next few years.

\section{References}

[1] L.A. Zadeh. Fuzzy sets. Inf. Control 8 (1965) 338-353.

[2] L.A. Zadeh. Outline of a new approach to analysis of complex systems and decision processes. IEEE Trans. Syst. Man Cybern. 3 (1973) 28-44.

[3] G. Klir and B. Yuan. Fuzzy Sets and Fuzzy Logic: Theory and Applications. Prentice Hall, Upper Saddle River, NJ, 1995.

[4] R. Sambuc. Function $\Phi$-Flous, Application a l'aide au Diagnostic en Pathologie Thyroidienne. These de Doctorat en Medicine. University of Marseille (1975).

[5] K.U. Jahn. Intervall-wertige Mengen. Math. Nachr. 68 (1975) 115-132.

[6] L.A. Zadeh. The concept of a linguistic variable and its application to approximate reasoning - I. Inf. Sci. 8 (1975) 199-249.

[7] I. Grattan-Guinness. Fuzzy membership mapped onto interval and many-valued quantities. Z. Math. Log. Grundl. Math. 22 (1976) 149-160.

[8] A. Dziech and M.B. Gorzalczany. Decision making in signal transmission problems with interval-valued fuzzy sets. Fuzzy Sets Syst. 23(2) (1987) 191-203.

[9] M.B. Gorzalczany. A method of inference in approximate reasoning based on interval-valued fuzzy sets. Fuzzy Sets Syst. 21 (1987) 1-17.

[10] M.B. Gorzalczany. Interval-valued fuzzy controller based on verbal model of object. Fuzzy Sets Syst. 28(1) (1988) 45-53.

[11] M.B. Gorzalczany. Interval-valued fuzzy inference involving uncertain (inconsistent) conditional propositions. Fuzzy Sets Syst. 29(2) (1989) 235-240.

[12] M.B. Gorzalczany. An interval-valued fuzzy inference method. Some basic properties. Fuzzy Sets Syst. 31(2) (1989) 243-251.

[13] I.B. Turksen. Interval valued fuzzy sets based on normal forms. Fuzzy Sets Syst. 20(2) (1986) 191-210.

[14] I.B. Turksen. Interval-valued fuzzy sets and compensatory AND. Fuzzy Sets Syst. 51 (1992) 295-307.

[15] I.B. Turksen and Z. Zhong. An approximate analogical reasoning schema based on similarity measures and interval-valued fuzzy sets. Fuzzy Sets Syst. 34 (1990) 323-346.

[16] I.B. Turksen and D.D. Yao. Representation of connectives in fuzzy reasoning: The view through normal forms. IEEE Trans. Syst. Man Cybern. 14 (1984) 191-210.

[17] J.M. Mendel. Advances in type-2 fuzzy sets and systems. Inf. Sci. 177 (2007) 84-110.

[18] D. Dubois. Foreword. In: H. Bustince, F. Herrera, and J. Montero (eds), Fuzzy Sets and Their Extensions: Representation, Aggregation and Models. Springer, New York, 2007.

[19] E. Trillas. Sobre funciones de negación en la teoría de conjuntos difusos. Stochastica III-1 (1979) 47-59 (in Spanish). English version in S. Barro, A. Sobrino, and A. Bugarin (eds), Advances of Fuzzy Logic. Universidad de Santiago de Compostela, 1998, pp. 31-43.

[20] J. Fodor and M. Roubens. Fuzzy Preference Modelling and Multicriteria Decision Support, Theory and Decision Library. Kluwer Academic, Dordrecht, 1994.

[21] P. Burillo and H. Bustince. Orderings in the referential set induced by an intuitionistic fuzzy relation. Notes IFS 1 (1995) 93-103.

[22] C. Cornelis, G. Deschrijver, and E.E. Kerre. Advances and challenges in interval-valued fuzzy logic. Fuzzy Sets Syst. 157 (2006) 622-627.

[23] G. Deschrijver and E.E. Kerre. On the relationship between some extensions of fuzzy set theory. Fuzzy Sets Syst. 133(2) (2003) 227-235. 
[24] G. Deschrijver. Arithmetic operators in interval-valued fuzzy set theory. Inf. Sci. 177(14) (2007) 2906-2924.

[25] J. Lazaro, T Calvo, and XAO Operators. The interval universe. In: Proceedings of 4th EUSFLAT 11th LFA, Barcelona, Spain, 2005, pp. 189-197.

[26] R.E. Moore. Interval Analysis. Prentice-Hall, Englewood Cliffs, NJ, 1966.

[27] N.S. Nedialkov, V. Kreinovich, and S.A. Starks. Interval arithmetic, affine arithmetic, Taylor series methods: Why, what next? Numer. Algo. 37 (1-4 SPEC. ISS) (2004) 325-336.

[28] Y.Y. Yao and J. Wang. Interval based uncertain reasoning using fuzzy and rough sets. In: P.P. Wang (ed), Advances in Machine Intelligence \& Soft Computing IV. Department of Electrical Engineering, Duke University, Durham, North Carolina, 1997, pp. 196-215.

[29] W. Zeng, Y. Shi, and H. Li. Representation theorem of interval-valued fuzzy set. Int. J. Uncertain. Fuzziness Knowl.-Based Syst. 14(3) (2006) 259-271.

[30] S. Jenei. A more efficient method for defining fuzzy connectives. Fuzzy Sets Syst. 90 (1997) 25-35.

[31] J.M. Mendel and R.I.J. Robert. Type-2 fuzzy sets made simple. IEEE Trans. Fuzzy Syst. 10(2) (2002) 117127.

[32] J.M. Mendel and H. Wu. Type-2 Fuzzistics for symmetric interval type-2 fuzzy sets: Part 1, forwarrd problems. IEEE Trans. Fuzzy Syst. 14(6) (2006) 781-792.

[33] J.M. Mendel. Uncertain Rule-Based Fuzzy Logic Systems. Prentice Hall, Upper Saddle River, NJ, 2001.

[34] K. Atanassov. Intuitionistic fuzzy sets. In: VIIth ITKR Session, Deposited in the Central Science and Technology Library of the Bulgarian Academy of Sciences, Sofia, Bulgaria, 1983, pp. 1684-1697.

[35] K. Atanassov. Intuitionistic Fuzzy Sets. Theory and Applications. Physica-Verlag, Heidelberg, 1999.

[36] D. Gómez, J. Montero, and H. Bustince. Sobre los conjuntos intuicionistas fuzzy de Atanassov. In: XIII Congreso Español sobre Tecnologías y Lógica Fuzzy, ESTYLF'06 (in Spanish), Ciudad Real, Spain, 2006, pp. 319-324.

[37] J. Montero, D. Gómez, and H. Bustince. On the relevance of some families of fuzzy sets. Fuzzy Sets Syst. 158(22) (2007) 2429-2442.

[38] E. Szmidt. Applications of Intuitionistic Fuzzy Sets in Decision Making. D.Sc. dissertation. Technical University of Sofia, 2000.

[39] I.K. Vlachos and G.D. Sergiadis. Inner product based entropy in the intuitionistic fuzzy setting. Int. J. Uncertain. Fuzziness Knowl.-Based Syst. 14(3) (2006) 351-367.

[40] W.L. Gau and D.J. Buehrer. Vague sets. IEEE Trans. Syst. Man Cybern. 23(2) (1993) 751-759.

[41] H. Bustince and P. Burillo. Vague sets are intuitionistic fuzzy sets. Fuzzy Sets Syst. 79 (1996) 403-405.

[42] G. Deschrijver and E.E. Kerre. On the position of intuitionistic fuzzy set theory in the framework of theories modelling imprecision. Inf. Sci. 177 (2007) 1860-1866.

[43] J.L. Deng. Introduction to grey system theory. J. Grey Syst. 1 (1989) 1-24.

[44] J.A. Goguen. L-fuzzy sets. J. Math. Anal. Appl. 18(1) (1967) 623-668.

[45] G.J. Wang and Y.Y. He. Intuitionistic fuzzy sets and L-fuzzy sets. Fuzzy Sets Syst. 110 (2000) 271-274.

[46] K. Hirota. Concepts of probabilistic sets. Fuzzy Sets Syst. 5 (1981) 31-46.

[47] K. Basu, R. Deb, and P.K. Pattanaik. Soft sets: An ordinal formulation of vagueness with some applications to the theory of choice. Fuzzy Sets Syst. 45 (1992) 45-58.

[48] I.B. Turksen. Fuzzy normal forms. Fuzzy Sets Syst. 69 (1995) 319-346.

[49] P. Burillo and H. Bustince. Construction theorems for intuitionistic fuzzy sets. Fuzzy Sets Syst. 84 (1996) 271-281.

[50] H.R. Tizhoosh. Image thresholding using type-2 fuzzy sets. Pattern Recognit. 38 (2005) 2363-2372.

[51] H. Bustince, V. Mohedano, E. Barrenechea, and M. Pagola. An algorithm for calculating the threshold of an image representing uncertainty through A-IFSs. In: Proceedings of 11th Information Processing and Management of Uncertainty in Knowledge-Based Systems, IPMU'06, Paris, France, 2006, pp. 2383-2390.

[52] H. Bustince, E. Barrenechea, M. Pagola, and R. Orduna. Image thresholding computation using Atanassov's intuitionistic fuzzy sets. J. Adv. Comput. Intell. Intell. Inf. 11(2) (2007) 187-194.

[53] H. Bustince, J. Kacprzyk, and V. Mohedano. Intuitionistic fuzzy generators. Application to intuitionistic fuzzy complementation. Fuzzy Sets Syst. 114 (2000) 485-504.

[54] E. Barrenechea. Image Processing with Interval-Valued Fuzzy Sets. Edge Detection. Contrast, Ph.D. Thesis. Universidad Publica de Navarra, 2005.

[55] F. Herrera and L. Martinez. A model based on linguistic 2-tuples for dealing with multigranularity hierachical linguistic context in multiexpert decision-making. IEEE Trans. Syst. Man Cybern. 31(2) (2001) 227-234.

[56] F. Liu, H. Geng, and Y.-Q. Zhang. Interactive fuzzy interval reasoning for smart web shopping. Appl. Soft Comput. 5(4) (2005) 433-439.

[57] R. Sepulveda, O. Castillo, P. Melin, A. Rodriguez-Diaz, and O. Montiel. Experimental study of intelligent controllers under uncertainty using type-1 and type-2 fuzzy logic. Inf. Sci. 177 (2007) 2023-2048. 
[58] A.M. Norwich and I.B. Turksen. The construction of membership functions. In: R.R. Yager (ed), Fuzzy Sets and Possibility Theory. Pergamon, New York, 1982, pp. 61-67.

[59] I.B. Turksen. Type 2 representation and reasoning for CWW. Fuzzy Sets Syst. 127 (2002) 17-36.

[60] H. Bustince. Indicator of inclusion grade for interval-valued fuzzy sets. Application to approximate reasoning based on interval-valued fuzzy sets. Int. J. Approx. Reason. 23(3) (2000) 137-209.

[61] P. Burillo and H. Bustince. Entropy on intuitionistic fuzzy sets and on interval-valued fuzzy sets. Fuzzy Sets Syst. 78 (1996) 305-316.

[62] H. Bustince, E. Barrenechea, and V. Mohedano. Intuitionistic fuzzy implication operators: An expression and main properties. Int. J. Uncertain. Fuzziness Knowl.-Based Syst. 12(3) (2004) 387-406.

[63] H. Bustince, V. Mohedano, E. Barrenechea, and M. Pagola. A study of the intuitionistic fuzzy S-implication operators. In: E. Herrera-Viedma (ed), Procesos de Toma de Decisiones, Modelado y Agregación de Preferencias (TIC-2002-11492-E), Granada, Spain, 2005, pp. 141-151.

[64] H. Bustince and P. Burillo. Interval-valued fuzzy relations in a set structure. J. Fuzzy Math. 4(4) (1996) 765785.

[65] H. Bustince and P. Burillo. Mathematical analysis of interval-valued fuzzy relations: Application to approximate reasoning. Fuzzy Sets Syst. 113 (2000) 205-219.

[66] H. Bustince and P. Burillo. Structures on intuitionistic fuzzy relations. Fuzzy Sets Syst. 78 (1996) 293-303.

[67] T. Bilgiç. Interval-valued preference structures. Eur. J. Oper. Res. 105 (1998) 162-183.

[68] O. Castillo and P. Melin. Fuzzy logic for plant monitoring and diagnostics. In: Proceedings of IEEE International Conference on Fuzzy Systems, Budapest, Hungary, 2004, pp. 25-29.

[69] J. Chiang. Fuzzy linear programming based on statistical confidence interval and interval-valued fuzzy set. Eur. J. Oper. Res. 129 (2001) 65-86.

[70] S.M. Chen, W.H. Hsiao, and W.T. Jong. Bidirectional approximate reasoning based on interval-valued fuzzy sets. Fuzzy Sets Syst. 91 (1997) 339-353.

[71] S.M. Chen. Measures of similarity between vague sets. Fuzzy Sets Syst. 74 (1995) 217-223.

[72] C. Cornelis, G. Deschrijver, and E. Kerre. Classification of intuitionistic fuzzy implicators: An algebraic approach. In: Proceedings of 6th Joint Conference on Information Sciences, Research Triangle Park, North Carolina, USA, 2002, pp. 105-108.

[73] C. Cornelis, G. Deschrijver, and E.E. Kerre. Implication in intuitionistic fuzzy and interval-valued fuzzy set theory: Construction, classification, application. Int. J. Approx. Reason. 35 (2004) 55-95.

[74] C. Cornelis, G. Deschrijver, and E. Kerre. Intuitionistic fuzzy connectives revisited. In: Proceedings of Information Processing and Management of Uncertainty in Knowledge-Based Systems, IPMU'02, Annecy, France, 2002, pp. 1839-1844.

[75] C. Cornelis and E.E. Kerre. Inclusion measures $\equiv$ uitionistic fuzzy set theory. Lect. Notes in Computer Science (Subseries LNAI), Vol. 2711. Springer, Nê York, 2003, pp. 345-356.

[76] S. Cubillo and E. Castineira. Contradiction in intuitionistic fuzzy sets. In: Proceedings of Information Processing and Management of Uncertainty in Knowledge-Based Systems, IPMU'04, Perugia, Italy, 2004, pp. 2180-2186.

[77] G. Deschrijver, C. Cornelis, and E.E. Kerre. On the representation of intuitionistic fuzzy T-norms and T-conorms. IEEE Trans. Fuzzy Syst. 12(1) (2004) 45-61.

[78] G. Deschrijver. The Archimedean property for t-norms in interval-valued fuzzy set theory. Fuzzy Sets Syst. 157 (2006) 2311-2327.

[79] G. Deschrijver and E.E. Kerre. Uninorms in L*-fuzzy set theory. Fuzzy Sets Syst. 148(2) (2004) 243-262.

[80] G. Deschrijver and E.E. Kerre. On the composition of intuitionistic fuzzy relations. Fuzzy Sets Syst. 136(3) (2003) 333-361.

[81] S. Dick, A. Schenker, W. Pedrycz, and A. Kandel. Regranulation: A granular algorithm enabling communication between granular worlds. Inf. Sci. 177 (2007) 408-435.

[82] D. Dubois and H. Prade. Fuzzy Sets and Systems: Theory and Applications. Academic Press, New York, 1980.

[83] E.P. Klement, R. Mesiar, and E. Pap. Triangular Norms. Kluwer, Dordrecht, 2002.

[84] E.P. Klement, R. Mesiar, and E. Pap. Triangular norms. Position paper I: Basic analytical and algebraic properties. Fuzzy Sets Syst. 143(1) (2004) 5-26.

[85] E.P. Klement, R. Mesiar, and E. Pap. Triangular norms. Position paper II: General constructions and parametrized families. Fuzzy Sets Syst. 145(3) (2004) 411-438.

[86] E.P. Klement, R. Mesiar, and E. Pap. Triangular norms. Position paper III: Continuous t-norms. Fuzzy Sets Syst. 145(3) (2004) 439-454.

[87] P. Smets and P. Magrez. Implication in fuzzy logic. Int. J. Approx. Reason. 1 (1987) 327-347.

[88] M. Mas, M. Monserrat, J. Torrens, and E. Trillas. A survey on fuzzy implication functions. IEEE Trans. Fuzzy Syst. 15(6) (2007) 1107-1121.

\section{AU: Pls. check Ref 75 if the location of the publisher is ok as inserted.}


[89] M. Mas, M. Monserrat, and J. Torrens. On two types of discrete implications. Int. J. Approx. Reason. 40(3) (2005) 262-279.

[90] P. Grzegorzewski. Distances between intuitionistic fuzzy sets and/or interval-valued fuzzy sets based on the Hausdorff metric. Fuzzy Sets Syst. 95(1) (1998) 113-117.

[91] E. Szmidt and J. Kacprzyk. Entropy for intuitionistic fuzzy sets. Fuzzy Sets Syst. 118(3) (2001) 467477.

[92] E. Szmidt and J. Kacprzyk. Entropy and similarity of intuitionistic fuzzy sets. In: Proceedings of Information Processing and Management of Uncertainty in Knowledge-Based Systems, IPMU'06, Paris, France, 2006, pp. 2375-2382.

[93] X. Liu. Entropy, distance measure and similarity measure of fuzzy sets and their relations. Fuzzy Sets Syst. 52 (1992) 305-318.

[94] H.B. Mitchell. Pattern recognition using type II fuzzy sets. Inf. Sci. 170 (2005) 409-418.

[95] H. Rezaei and M. Mukaidono. New similarity measures of intuitionistic fuzzy sets. J. Adv. Comput. Intell. Intell. Inf. 11(2) (2007) 202-209.

[96] I.K. Vlachos and G.D. Sergiadis. Subsethood, entropy, and cardinality for interval-valued fuzzy sets an algebraic derivation. Fuzzy Sets Syst. 158 (2007) 1384-1396.

[97] C. Zhang and H. Fu. Similarity measure on three kinds of fuzzy sets. Pattern Recognit. Lett. 27(12) (2006) $1307-1317$.

[98] W. Zeng and H. Li. Relationship between similarity measures and entropy of interval valued fuzzy sets. Fuzzy Sets Syst. 157(11) (2006) 1477-1484.

[99] P. Grzegorzewski and E. Mrowka. On the entropy of intuitionistic fuzzy sets and interval-valued fuzzy sets. In: Proceedings of 10th International Conference on Information Processing and Management of Uncertainty in Knowledge-Based Systems, IPMU'04, Perugia, Italy, 2004, pp. 1419-1426.

[100] W.-L. Hung and M.-S. Yang. Similarity measures of intuitionistic fuzzy sets based on Hausdorff metric. Pattern Recognit. Lett. 25 (2004) 1603-1611.

[101] D. Li and C. Cheng. New similarity measures of intuitionistic fuzzy sets and application to pattern recognition. Pattern Recognit. Lett. 23 (2002) 221-225.

[102] Z. Liang and P. Shi. Similarity measures on intuitionistic fuzzy sets. Pattern Recognit. Lett. 24 (2003) 26872693.

[103] H.B. Mitchell. On the Dengfeng-Chuntian similarity measure and its application to pattern recognition. Pattern Recognit. Lett. 24 (2003) 3101-3104.

[104] E. Szmidt and J. Kacprzyk. Similarity of intuitionistic fuzzy sets and the Jaccard coefficient. In: Proceedings of 10th International Conference on Information Processing and Management of Uncertainty in Knowledge-Based Systems, IPMU'04, Perugia, Italy, 2004, pp. 1405-1412.

[105] E. Szmidt, J. Kacprzyk. A measure of similarity for intuitionistic fuzzy sets. In: Proceedings of 3th International Conference in Fuzzy Logic and Technology, Zittau, Germany, 2003, pp. 206-209.

[106] G.-J. Wang and X.-P. Li. On the IV-fuzzy degree and the IV-similar degree of IVFS and their integral representation. J. Eng. Math. 21 (2004) 195-201.

[107] A. Kehagias and M. Konstantinidou. L-fuzzy valued inclusion measure, L-fuzzy similarity and L-fuzzy distance. Fuzzy Sets Syst. 136 (2003) 313-332.

[108] T. Gerstenkorn and J. Manko. Correlation of intuitionistic fuzzy sets. Fuzzy Sets Syst. 44 (1991) 39-43.

[109] G. Wang and X. Li. Correlation and information energy of interval-valued fuzzy numbers. Fuzzy Sets Syst. 103 (1999) 169-175.

[110] I.B. Turksen. Interval-valued strict preference with Zadeh triples. Fuzzy Sets Syst. 78 (1996) 183-195.

[111] L.J. Kohout and W. Bandler. Fuzzy interval inference utilizing the checklist paradigm and BK-relational products. In: R.B. Kearfort and V. Kreinovich (eds), Application of Interval Computations. Kluwer, Dordrecht, 1996, pp. 291-335.

[112] L.A. Zadeh. Theory of approximate reasoning. In: J. Hayes, D. Michie, and L.I. Mikulich (eds), Machine Intelligence. Halstead Press, New York, 1979, pp. 149-194.

[113] M.K. Roy and R. Biswas. I-v fuzzy relations and Sanchez's approach for medical diagnosis. Fuzzy Sets Syst. 47 (1992) 35-38.

[114] M. Wagenknecht. On transitive solutions of fuzzy equations, inequalities and lower approximations of fuzzy relations. Fuzzy Sets Syst. 75(2) (1995) 229-240.

[115] W. Pedrycz. Processing in relational structures: Fuzzy relational equations. Fuzzy Sets Syst. 40(1) (1991) 77106.

[116] T. Arnauld and S. Tano. Interval-valued fuzzy backward reasoning. IEEE Trans. Fuzzy Syst. 3(4) (1995) 425437. 
[117] S. Fukami, M. Mizumoto, and K. Tanaka. Some considerations on fuzzy conditional inference. Fuzzy Sets Syst. 4 (1980) 243-273.

[118] J.F. Baldwin, B.W. Pilsworth. Axiomatic approach to implication for approximate reasoning with fuzzy logic. Fuzzy Sets Syst. 3 (1980) 193-219.

[119] A. Sala, B. Tormos, V. Maciàn, and E. Royo. Fuzzy diagnosis module based on interval fuzzy logic: Oil analysis application. In: Proceedings of the International Conference on Informatics in Control, Automation and Robotics, ICINCO 2005, Barcelona, Spain, 2005, pp. 85-90.

[120] S. Wang, F.-L. Chung, Y.Y. Li, D. Hu, and X.S. Wu. A new Gaussian noise filter based on interval type-2 fuzzy logic systems. Soft Comput. 9(5) (2005) 398-406.

[121] L.A. Zadeh. From computing with numbers to computing with words - from manipulation of measurements to manipulation of perceptions. IEEE Trans. Circuits Syst. 4 (1999) 105-119.

[122] I.B. Turksen. Meta-linguistic axioms as foundation for computing with words. Inf. Sci. 177 (2006) 332-359.

[123] J.M. Mendel. Computing with words and its relationships with fuzzistics. Inf. Sci. 177 (2007) 988-1006.

[124] F. Herrera, L. Martinez, and P.J. Sanchez. Managing non-homogeneous information in group decision making. Eur. J. Oper. Res. 166(1) (2005) 115-132.

[125] L. Martinez, J. Liu, Da Ruan, and J-B. Yang. Dealing with heterogeneous information in engineering evaluation processes. Inf. Sci. 177 (2007) 1533-1542.

[126] A. Pankowska and M. Wygralak. On hesitation degrees in IF-set theory. In: L. Rutkowski, J. Siekmann, and R. Ta $\equiv$ wicz (eds), Artificial Intelligence and Soft Computing, Lecture Notes in Artificial Intelligence, Vol. 3070, 2004, pp. 338-343.

[127] A. Pankowska and M. Wygralak. General IF-sets with triangular norms and their applications to group decision making. Inf. Sci. 176 (2006) 2713-2754.

[128] E. Szmidt and J. Kacprzyk. Group decision making under intuitionistic fuzzy preference relations. In: Proceedings of Information Processing and Management of Uncertainty in Knowledge-Based Systems, IPMU'98, Paris, France, 1998, pp. 172-178.

[129] H.-F. Wang and M.-L. Wang. A fuzzy multiobjective linear programming. Fuzzy Sets Syst. 86(1) (1997) 61-72.

[130] A. Serguieva and J. Hunter. Fuzzy interval methods in investment risk appraisal. Fuzzy Sets Syst. 142(3) (2004) 443-466.

[131] J.S. Yao and T.S. Shih. Fuzzy revenue for fuzzy demand quantity based on interval-valued fuzzy sets. Comput. Oper. Res. 29 (2002) 1495-1535.

[132] H. Hagras. A hierarchical type-2 fuzzy logic control architecture for autonomous mobile robots. IEEE Trans. Fuzzy Syst. 12(4) (2004) 524-539.

[133] H.T. Nguyen, V. Kreinovich, R.N. Lea, and D. Tolbert. How to control if even experts are not sure: Minimizing intervals of uncertainty. In: Abstracts of Workshop on Interval Methods, International Conference on Interval Computations, Lafayette, Louisiana, 1993, p. 27.

[134] K.C. Wu. Fuzzy interval control of mobile robots. Comput. Electr. Eng. 22(3) (1996) 211-229.

[135] W. Pedrycz. Fuzzy modelling: Fundamentals, construction and evaluation. Fuzzy Sets Syst. 41(1) (1991) 1-15.

[136] W. Pedrycz. Relevancy of fuzzy models. Inf. Sci. 52(3) (1990) 285-302.

[137] W. Pedrycz. Direct and inverse problem in comparison of fuzzy data. Fuzzy Sets Syst. 34(2) (1990) 223-235.

[138] L.A. Zadeh. Fuzzy sets and information granularity. In: M. Gupta, R. Ragade, and R. Yager (eds), Advances in Fuzzy Set Theory and Applications. North-Holland, Amsterdam, 1979, pp. 3-18.

[139] L.A. Zadeh. Towards a theory of fuzzy information granulation and its centrality in human reasoning and fuzzy logic. Fuzzy Sets Syst. 19 (1996) 103-111.

[140] Y.Y. Yao. Granular Computing: basic issues and possible solutions. In: Proceedings of 5th Joint Conference on Information Sciences, Cary, North Carolina, USA, 2000, pp. 186-189.

[141] A. Bargiela, W. Pedrycz. Granular Computing: An Introduction. Kluwer Academic Publishers, Boston, MA, 2003. 


\begin{abstract}
In this chapter we carry out a survey of the theoretical properties and the most important applications of interval-valued fuzzy sets (IVFSs). We present the conditions under which IVFSs should be used and study their relation with other extensions of fuzzy set theory. We also indicate the three major problems of this theory from our point of view. We study structural properties and show the connectives for operating. We review the most commonly used numerical measures of information and pose the problem of the loss of information when they are not defined in an intervalar way. Next we show the importance of these sets in some applications, as, for example, in the thresholding of certain types of images as well as in approximate reasoning. Lastly, we describe their connection with granular computing and present a comprehensive bibliography. Throughout the chapter we list different open problems.
\end{abstract}

Keywords: interval-valued fuzzy sets; interval type 2 fuzzy sets; IV negation; IV $t$-norms; IV $t$-conorm; interval-valued fuzzy implication operator; indetermination index; IV distance; degree of compatibility; IV entropy; IV similarity; IV inclusion measure 